\title{
Transcendental Morse inequality and generalized Okounkov bodies
}

\author{
Ya Deng
}

\begin{abstract}
The main goal of this article is to construct "arithmetic Okounkov bodies" for an arbitrary pseudo-effective $(1,1)$-class $\alpha$ on a Kähler manifold. First, we prove the differentiability of volumes of big classes for Kähler manifolds on which modified nef cones and nef cones coincide. As a consequence, we prove Demailly's transcendental Morse inequality for these particular Kähler manifolds. In the second part, we construct the generalized Okounkov body for any big $(1,1)$-class, and prove that it coincides with the Okounkov body when the big class is rational. Next, we give a complete characterization of generalized Okounkov bodies on surfaces, and relate the standard Euclidean volume of the body to the volume of the corresponding big class as defined by Boucksom; this solves a problem raised by Lazarsfeld and Mustaţă in the case of surfaces. Finally, we study the behavior of the generalized Okounkov bodies on the boundary of the big cones.
\end{abstract}

\section{Introduction}

In [Oko96] Okounkov introduced a natural procedure to associate a convex body $\Delta(D)$ in $\mathbb{R}^{n}$ with any ample divisor $D$ on an $n$-dimensional projective variety. Relying on the work of Okounkov, Lazarsfeld and Mustaţă [LM09] and Kaveh and Khovanskii [KK12a, KK12b] have systematically studied Okounkov's construction, and associated with any big divisor and any fixed flag of subvarieties a convex body which is now called the Okounkov body.

We briefly recall the construction of the Okounkov body. We start with a complex projective variety $X$ of dimension $n$. Fix a flag

$$
Y_{\bullet}: X=Y_{0} \supset Y_{1} \supset Y_{2} \supset \cdots \supset Y_{n-1} \supset Y_{n}=\{p\},
$$

where $Y_{i}$ is a smooth irreducible subvariety of codimension $i$ in $X$. For a given big divisor $D$, one defines a valuation-like function

$$
\mu=\mu_{Y_{\bullet}, D}:\left(H^{0}\left(X, \mathcal{O}_{X}(D)\right)-\{0\}\right) \rightarrow \mathbb{Z}^{n}
$$

as follows. First, set $\mu_{1}=\mu_{1}(s)=\operatorname{ord}_{Y_{1}}(s)$. Dividing $s$ by a local equation of $Y_{1}$, we obtain

Received 17 April 2015, accepted in final form 12 May 2016.

2010 Mathematics Subject Classification 32J25 (primary), 32J27, 32J15 (secondary).

Keywords: generalized Okounkov body, positive current, Siu decomposition, divisorial Zariski decomposition, Lelong number, transcendental Morse inequality, numerical dimension, differentiability formula.

This journal is (c) Foundation Compositio Mathematica 2017. This article is distributed with Open Access under the terms of the Creative Commons Attribution Non-Commercial License, which permits non-commercial reuse, distribution, and reproduction in any medium, provided that the original work is properly cited. For commercial re-use, please contact the Foundation Compositio Mathematica.

The author is supported by the China Scholarship Council and by the ERC ALKAGE project. 


\section{Y. DENG}

a section

$$
\widetilde{s}_{1} \in H^{0}\left(X, \mathcal{O}_{X}\left(D-\mu_{1} Y_{1}\right)\right)
$$

that does not vanish identically along $Y_{1}$. We restrict $\widetilde{s}_{1}$ on $Y_{1}$ to get a non-zero section

$$
s_{1} \in H^{0}\left(Y_{1}, \mathcal{O}_{Y_{1}}\left(D-\mu_{1} Y_{1}\right)\right) \text {; }
$$

then we write $\mu_{2}(s)=\operatorname{ord}_{Y_{2}}\left(s_{1}\right)$ and continue in this fashion to define the remaining integers $\mu_{i}(s)$. The image of the function $\mu$ in $\mathbb{Z}^{n}$ is denoted by $\mu(D)$. With this in hand, we define the Okounkov body of $D$ with respect to the fixed flag $Y_{\bullet}$ to be

$$
\Delta(D)=\Delta_{Y_{\bullet}}(D)=\text { closed convex hull }\left(\bigcup_{m \geqslant 1} \frac{1}{m} \cdot \mu(m D)\right) \subseteq \mathbb{R}^{n} .
$$

According to the open question raised in the final part of [LM09], it is quite natural to wonder whether one can construct "arithmetic Okounkov bodies" for an arbitrary pseudo-effective $(1,1)$ class $\alpha$ on a Kähler manifold and realize the volumes of these classes by convex bodies as well. In our paper, using positive currents in a natural way, we give a construction of a convex body $\Delta(\alpha)$ associated with such a class $\alpha$, and show that this newly defined convex body coincides with the Okounkov body when $\alpha \in \mathrm{NS}_{\mathbb{R}}(X)$.

TheOrem 1.1. Let $X$ be a smooth projective variety of dimension $n$, let $L$ be a big line bundle on $X$, and let $Y$ • be a fixed admissible flag. Then we have

$$
\Delta\left(c_{1}(L)\right)=\Delta(L)=\overline{\bigcup_{m=1}^{\infty} \frac{1}{m} \nu(m L)} .
$$

Moreover, in the definition of the Okounkov body $\Delta(L)$, it suffices to take the closure of the set of normalized valuation vectors instead of the closure of the convex hull.

By Theorem 1.1, we know that our definition of the Okounkov body for any pseudo-effective class could be treated as a generalization of the original Okounkov body. A very interesting problem is to find out exactly which points in the Okounkov body $\Delta(L)$ are given by valuations of sections. This is expressed by saying that a rational point of $\Delta(L)$ is "valuative". By Theorem 1.1, we can give some partial answers to this question, which have been given in [KL14] in the case of surfaces.

Corollary 1.2. Let $X$ be a projective variety of dimension $n$, and let $Y \bullet$ be an admissible flag. If $L$ is a big line bundle, then any rational point in $\operatorname{int}(\Delta(L))$ is a valuative point.

It is quite natural to wonder whether our newly defined convex body for big classes behaves similarly to the original Okounkov body. In the situation of complex surfaces, we give an affirmative answer to the question raised in [LM09], as follows.

Theorem 1.3. Let $X$ be a compact Kähler surface, and let $\alpha \in H^{1,1}(X, \mathbb{R})$ be a big class. If $C$ is an irreducible divisor of $X$, there are piecewise linear continuous functions

$$
f, g:[a, s] \mapsto \mathbb{R}_{+}
$$

with $f$ convex, $g$ concave, and $f \leqslant g$, such that $\Delta(\alpha) \subset \mathbb{R}^{2}$ is the region bounded by the graphs of $f$ and $g$ :

$$
\Delta(\alpha)=\left\{(t, y) \in \mathbb{R}^{2} \mid a \leqslant t \leqslant s, \text { and } f(t) \leqslant y \leqslant g(t)\right\}
$$




\section{Generalized OKOUNKov Bodies}

Here $\Delta(\alpha)$ is the generalized Okounkov body with respect to the fixed flag

$$
X \supseteq C \supseteq\{x\}
$$

and $s=\sup \{t>0 \mid \alpha-t C$ is big $\}$. If $C$ is nef, then $a=0$ and $f(t)$ is increasing; otherwise, $a=\sup \left\{t>0 \mid C \subseteq E_{n K}(\alpha-t C)\right\}$, where $E_{n K}:=\bigcap_{T} E_{+}(T)$ for $T$ ranging over the Kähler currents in $\alpha$, which is the non-Kähler locus. Moreover, $\Delta(\alpha)$ is a finite polygon whose number of vertices is bounded by $2 \rho(X)+2$, where $\rho(X)$ is the Picard number of $X$, and

$$
\operatorname{vol}_{X}(\alpha)=2 \operatorname{vol}_{\mathbb{R}^{2}}(\Delta(\alpha)) .
$$

In [LM09], it was asked whether the Okounkov body of a divisor on a complex surface could be an infinite polygon. In [KLM12], it was shown that the Okounkov body is always a finite polygon. Here we give an explicit description for the "finiteness" of the polygons appearing as generalized Okounkov bodies of big classes and conclude that the finiteness also holds for the original Okounkov bodies by Theorem 1.1.

As one might suspect from the construction of Okounkov bodies, the Euclidean volume of $\Delta(D)$ has a strong connection with the growth of the groups $H^{0}\left(X, \mathcal{O}_{X}(m D)\right)$. In [LM09], the following precise relations were shown:

$$
n ! \cdot \operatorname{vol}_{\mathbb{R}^{n}}(\Delta(D))=\operatorname{vol}_{X}(D):=\lim _{k \rightarrow \infty} \frac{n !}{k^{n}} h^{0}\left(X, \mathcal{O}_{X}(k D)\right) .
$$

The proof of (1.1) relies on properties of sub-semigroups of $\mathbb{N}^{n+1}$ constructed from the graded linear series $\left\{H^{0}\left(X, \mathcal{O}_{X}(m D)\right)\right\}_{m \geqslant 0}$. However, when $\alpha$ is a big class which does not belong to $\mathrm{NS}_{\mathbb{R}}(X)$, there are no such algebraic objects which correspond to $\operatorname{vol}_{X}(\alpha)$, and we only have the following analytic definition due to Boucksom ([Bou02]):

$$
\operatorname{vol}_{X}(\alpha):=\sup _{T} \int_{X} T_{a c}^{n},
$$

where $T$ ranges over all positive $(1,1)$-currents. Therefore, it is quite natural to propose the following conjecture.

ConjeCture 1.4. Let $X$ be a compact Kähler manifold of dimension $n$. For any big class $\alpha \in H^{1,1}(X, \mathbb{R})$, we have

$$
\operatorname{vol}_{\mathbb{R}^{n}}(\Delta(\alpha))=\frac{1}{n !} \cdot \operatorname{vol}_{X}(\alpha) .
$$

In Theorem 1.3, we prove this conjecture in dimension 2. Our method is to relate the Euclidean volume of the slice of the generalized Okounkov body to the differential of the volume of the big class. We prove the following differentiability formula for volumes of big classses.

TheOREm 1.5. Let $X$ be a compact Kähler surface, and let $\alpha$ be a big class. If $\beta$ is a nef class or $\beta=\{C\}$, where $C$ is an irreducible curve, we have

$$
\left.\frac{d}{d t}\right|_{t=0} \operatorname{vol}_{X}(\alpha+t \beta)=2 Z(\alpha) \cdot \beta
$$

where $Z(\alpha)$ is the divisorial Zariski decomposition of $\alpha$ defined in Section 2.6.

A direct corollary of this formula is the transcendental Morse inequality.

Theorem 1.6. Let $X$ be a compact Kähler surface. If $\alpha$ and $\beta$ are nef classes satisfying the inequality $\alpha^{2}-2 \alpha \cdot \beta>0$, then $\alpha-\beta$ is big and $\operatorname{vol}_{X}(\alpha-\beta) \geqslant \alpha^{2}-2 \alpha \cdot \beta$. 


\section{Y. DENG}

In higher dimension, we also have a differentiability formula for big classes on some special Kähler manifolds.

THEOREM 1.7. Let $X$ be a compact Kähler manifold of dimension $n$ on which the modified nef cone $\mathcal{M N}$ coincides with the nef cone $\mathcal{N}$. If $\alpha \in H^{1,1}(X, \mathbb{R})$ is a big class and $\beta \in H^{1,1}(X, \mathbb{R})$ is a nef class, then

$$
\operatorname{vol}_{X}(\alpha+\beta)=\operatorname{vol}_{X}(\alpha)+n \int_{0}^{1} Z(\alpha+t \beta)^{n-1} \cdot \beta \mathrm{d} t .
$$

As a consequence, $\operatorname{vol}_{X}(\alpha+t \beta)$ is $\mathcal{C}^{1}$ for $t \in \mathbb{R}^{+}$, and we have

$$
\left.\frac{d}{d t}\right|_{t=t_{0}} \operatorname{vol}_{X}(\alpha+t \beta)=n Z\left(\alpha+t_{0} \beta\right)^{n-1} \cdot \beta
$$

for $t_{0} \geqslant 0$.

Finally, we study the generalized Okounkov bodies for pseudo-effective classes in Kähler surfaces. We summarize our results as follows.

Theorem 1.8. Let $X$ be a Kähler surface, and let $\alpha$ be any pseudo-effective but not big class.

(i) If the numerical dimension $n(\alpha)$ is 0 , then for any irreducible curve $C$ which is not contained in the negative part $N(\alpha)$, we have the generalized Okounkov body

$$
\Delta_{(C, x)}(\alpha)=0 \times \nu_{x}\left(\left.N(\alpha)\right|_{C}\right),
$$

where $\nu_{x}\left(\left.N(\alpha)\right|_{C}\right)=\nu\left(\left.N(\alpha)\right|_{C}, x\right)$ is the Lelong number of $N(\alpha)$ at $x$.

(ii) If $n(\alpha)=1$, then for any irreducible curve $C$ satisfying $Z(\alpha) \cdot C>0$, we have

$$
\Delta_{(C, x)}(\alpha)=0 \times\left[\nu_{x}\left(\left.N(\alpha)\right|_{C}\right), \nu_{x}\left(\left.N(\alpha)\right|_{C}\right)+Z(\alpha) \cdot C\right] .
$$

In particular, the numerical dimension determines the dimension of the generalized Okounkov body.

\section{Technical preliminaries}

\subsection{Siu decomposition}

Let $T$ be a closed positive current of bidegree $(p, p)$ on a complex manifold $X$. We denote by $\nu(T, x)$ its Lelong number at a point $x \in X$. For any $c>0$, the Lelong upperlevel sets are defined by

$$
E_{c}(T):=\{x \in X, \nu(T, x) \geqslant c\} .
$$

In [Siu74], Siu proved that $E_{c}(T)$ is an analytic subset of $X$, of codimension at least $p$. Moreover, $T$ can be written as a convergent series of closed positive currents

$$
T=\sum_{k=1}^{+\infty} \nu\left(T, Z_{k}\right)\left[Z_{k}\right]+R,
$$

where $\left[Z_{k}\right]$ is a current of integration over an irreducible analytic set of dimension $p$, and $R$ is a residual current with the property that $\operatorname{dim} E_{c}(R)<p$ for every $c>0$. This decomposition is locally and globally unique: the sets $Z_{k}$ are precisely the $p$-dimensional components occurring in the upperlevel sets $E_{c}(T)$, and $\nu\left(T, Z_{k}\right):=\inf \left\{\nu(T, x) \mid x \in Z_{k}\right\}$ is the generic Lelong number of $T$ along $Z_{k}$. 


\section{Generalized OKOUNKov Bodies}

\subsection{Currents with analytic singularities}

A closed positive $(1,1)$-current $T$ on a compact complex manifold $X$ is said to have analytic (respectively, algebraic) singularities along a subscheme $V(\mathcal{I})$ defined by an ideal $\mathcal{I}$ if there exists some $c \in \mathbb{R}_{>0}$ (respectively, $c \in \mathbb{Q}_{>0}$ ) such that, locally, we have

$$
T=\frac{c}{2} d d^{c} \log \left(\left|f_{1}\right|^{2}+\cdots+\left|f_{k}\right|^{2}\right)+d d^{c} v,
$$

where $f_{1}, \ldots, f_{k}$ are local generators of $\mathcal{I}$ and $v \in L_{\text {loc }}^{\infty}$. In the algebraic case, we have the additional condition that $X$ and $V(\mathcal{I})$ be algebraic. Moreover, if $v$ is smooth, $T$ will be said to have mild analytic singularities. In these situations, we call the sum $\sum \nu(T, D) D$ which appears in the Siu decomposition of $T$ the divisorial part of $T$. Using the Lelong-Poincaré formula, it is straightforward to check that the divisorial part $\sum \nu(T, D) D$ of a closed $(1,1)$-current $T$ with analytic singularities along the subscheme $V(\mathcal{I})$ is just the divisorial part of $V(\mathcal{I})$, times the constant $c>0$ appearing in the definition of analytic singularities. The residual part $R$ has analytic singularities in codimension at least 2 . If we set $E_{+}(T):=\{x \in X \mid \nu(T, x)>0\}$, then $E_{+}(T)$ is exactly the support of $V(\mathcal{I})$. Moreover, if $V \nsubseteq E_{+}(T)$ for some smooth variety $V$, then $\left.T\right|_{V}:=\left.\frac{c}{2} d d^{c} \log \left(\left|f_{1}\right|^{2}+\cdots+\left|f_{k}\right|^{2}\right)\right|_{V}+\left.d d^{c} v\right|_{V}$ is well defined, for $\left|f_{1}\right|^{2}+\cdots+\left|f_{k}\right|^{2}$ and $v$ are not identically equal to $-\infty$ on $V$. It is easy to check that this definition does not depend on the choice of the local potential of $T$.

Definition 2.1. If $\alpha \in H_{\partial \bar{\partial}}^{1,1}(X, \mathbb{R})$ is a big class, we define its non-Kähler locus as $E_{n K}:=$ $\bigcap_{T} E_{+}(T)$ for $T$ ranging over the Kähler currents in $\alpha$.

We will often use the following theorem due to Collins and Tosatti.

Theorem 2.2 ([CT15]). Let $X$ be a compact Kähler manifold of dimension $n$. Given a nef and big class $\alpha$, we define a subset of $X$ which measures its non-Kählerianity, namely the null locus

$$
\operatorname{Null}(\alpha):=\bigcup_{\int_{V}} \alpha_{\operatorname{dim} V=0} V
$$

where the union is taken over all positive-dimensional irreducible analytic subvarieties of $X$. Then we have

$$
\operatorname{Null}(\alpha)=E_{n K}(\alpha)
$$

\subsection{Regularization of currents}

We will need Demailly's regularization theorem [Dem92] for closed (1,1)-currents, which enables us to approximate a given current by currents with analytic singularities, with a loss of positivity that is arbitrarily small. In particular, we could approximate a Kähler current $T$ inside its cohomology class by Kähler currents $T_{k}$ with algebraic singularities, with a good control of the singularities. A big class therefore contains plenty of Kähler currents with analytic singularities.

THEOREM 2.3. Let $T$ be a closed almost positive (1,1)-current on a compact complex manifold $X$, and fix a Hermitian form $\omega$. Suppose $T \geqslant \gamma$ for some real $(1,1)$-form $\gamma$ on $X$. Then there exists a sequence $T_{k}$ of currents with algebraic singularities in the cohomology class $\{T\}$ which converges weakly to $T$, such that $T_{k} \geqslant \gamma-\epsilon_{k} \omega$ for some sequence $\epsilon_{k}>0$ decreasing to 0 , and $\nu\left(T_{k}, x\right)$ increases to $\nu(T, x)$ uniformly with respect to $x \in X$. 


\section{Y. DENG}

\subsection{Currents with minimal singularities}

Let $T_{1}=\theta_{1}+d d^{c} \varphi_{1}$ and $T_{2}=\theta_{2}+d d^{c} \varphi_{2}$ be two closed almost positive (1,1)-currents on $X$, where the $\theta_{i}$ are smooth forms and the $\varphi_{i}$ are almost pluri-subharmonic functions. We say that $T_{1}$ is less singular than $T_{2}$ (and write $T_{1} \preceq T_{2}$ ) if we have $\varphi_{2} \leqslant \varphi_{1}+C$ for some constant $C$.

Let $\alpha$ be a class in $H_{\partial \bar{\partial}}^{1,1}(X, \mathbb{R})$, and let $\gamma$ be a smooth real $(1,1)$-form. We denote by $\alpha[\gamma]$ the set of closed almost positive (1,1)-currents $T \in \alpha$ with $T \geqslant \gamma$. Since the set of potentials of such currents is stable under taking a supremum, we conclude by standard pluripotential theory that there exists a closed almost positive $(1,1)$-current $T_{\min , \gamma} \in \alpha[\gamma]$ which has minimal singularities in $\alpha[\gamma]$. The current $T_{\min , \gamma}$ is well defined modulo $d d^{c} L^{\infty}$. For each $\epsilon>0$, denote by $T_{\min , \epsilon}=T_{\min , \epsilon}(\alpha)$ a current with minimal singularities in $\alpha[-\omega]$, where $\omega$ is some reference Hermitian form. The minimal multiplicity at $x \in X$ of the pseudo-effective class $\alpha \in H_{\partial \bar{\partial}}^{1,1}(X, \mathbb{R})$ is defined as

$$
\nu(\alpha, x):=\sup _{\epsilon>0} \nu\left(T_{\min , \epsilon}, x\right) .
$$

For a prime divisor $D$, we define the generic minimal multiplicity of $\alpha$ along $D$ as

$$
\nu(\alpha, D):=\inf \{\nu(\alpha, x) \mid x \in D)\} .
$$

We then have $\nu(\alpha, D)=\sup _{\epsilon>0} \nu\left(T_{\min , \epsilon}, D\right)$.

\subsection{Lebesgue decomposition}

A current $T$ can be locally seen as a form with distribution coefficients. When $T$ is positive, the distributions are positive measures which admit a Lebesgue decomposition into an absolutely continuous part (with respect to the Lebesgue measure on $X$ ) and a singular part. Therefore we obtain the decomposition $T=T_{\mathrm{ac}}+T_{\text {sing }}$, with $T_{\mathrm{ac}}$ and $T_{\text {sing }}$ globally determined thanks to the uniqueness of the Lebesgue decomposition.

Now, we assume that $T$ is a $(1,1)$-current. The absolutely continuous part $T_{\mathrm{ac}}$ is considered as a $(1,1)$-form with $L_{\text {loc }}^{1}$ coefficients, and, more generally, we have $T_{\text {ac }} \geqslant \gamma$ whenever $T \geqslant \gamma$ for some real smooth real form $\gamma$. Thus, we can define the product $T_{\mathrm{ac}}^{k}$ of $k$ copies of $T_{\mathrm{ac}}$ almost everywhere. This yields a positive Borel $(k, k)$-form.

\subsection{Modified nef cone and divisorial Zariski decomposition}

In this subsection, we collect some definitions and properties of the modified nef cone and divisorial Zariski decomposition. See [Bou04] for more details.

Definition 2.4. Let $X$ be compact complex manifold, and let $\omega$ be some reference Hermitian form. Let $\alpha$ be a class in $H_{\partial \bar{\partial}}^{1,1}(X, \mathbb{R})$.

(i) The class $\alpha$ is said to be a modified Kähler class if and only if it contains a Kähler current $T$ with $\nu(T, D)=0$ for all prime divisors $D$ in $X$.

(ii) The class $\alpha$ is said to be a modified nef class if and only if, for every $\epsilon>0$, there exists a closed $(1,1)$-current $T_{\epsilon}$ with $T_{\epsilon} \geqslant-\epsilon \omega$ and $\nu\left(T_{\epsilon}, D\right)=0$ for every prime $D$.

Remark 2.5. The modified nef cone $\mathcal{M N}$ is a closed convex cone which contains the nef cone $\mathcal{N}$. When $X$ is a Kähler manifold, $\mathcal{M N}$ is just the interior of the modified Kähler cone $\mathcal{M K}$.

Remark 2.6. For a complex surface, the Kähler (respectively, nef) cone and the modified Kähler (respectively, modified nef) cone coincide. Indeed, analytic singularities in codimension 2 of a Kähler current $T$ are just isolated points. Therefore, the class $\{T\}$ is a Kähler class. 


\section{Generalized Okounkov Bodies}

Definition 2.7. The negative part of a pseudo-effective class $\alpha \in H_{\partial \bar{\partial}}^{1,1}(X, \mathbb{R})$ is defined as $N(\alpha):=\sum \nu(\alpha, D) D$. The Zariski projection of $\alpha$ is $Z(\alpha):=\alpha-\{N(\alpha)\}$. We call the decomposition $\alpha=Z(\alpha)+\{N(\alpha)\}$ the divisorial Zariski decomposition of $\alpha$.

Remark 2.8. We claim that the volume of $Z(\alpha)$ is equal to the volume of $\alpha$. Indeed, if $T$ is a positive current in $\alpha$, then we have $T \geqslant N(\alpha)$ since $T \in \alpha[-\epsilon \omega]$ for each $\epsilon>0$, and we conclude that $T \mapsto T-N(\alpha)$ is a bijection between the positive currents in $\alpha$ and those in $Z(\alpha)$. Furthermore, we notice that $(T-N(\alpha))_{\mathrm{ac}}=T_{\mathrm{ac}}$, and thus by the definition of the volume of the pseudo-effective classes we conclude that $\operatorname{vol}_{X}(\alpha)=\operatorname{vol}_{X}(Z(\alpha))$.

Definition 2.9. (i) A family $D_{1}, \ldots, D_{q}$ of prime divisors is said to be an exceptional family if and only if the convex cone generated by the cohomology classes of the $D_{i}$ meets the modified nef cone at 0 only.

(ii) An effective $\mathbb{R}$-divisor $E$ is said to be exceptional if and only if its prime components constitute an exceptional family.

We have the following properties of exceptional divisors.

Theorem 2.10. (i) An effective $\mathbb{R}$-divisor $E$ is exceptional if and only if $Z(E)=0$.

(ii) If $E$ is an exceptional effective $\mathbb{R}$-divisor, we have $E=N(\{E\})$.

(iii) If $D_{1}, \ldots, D_{q}$ is an exceptional family of primes, then the classes $\left\{D_{1}\right\}, \ldots,\left\{D_{q}\right\}$ are linearly independent in $\mathrm{NS}_{\mathbb{R}}(X) \subset H^{1,1}(X, \mathbb{R})$. In particular, the lengths of the exceptional families of primes are uniformly bounded by the Picard number $\rho(X)$.

(iv) Let $X$ be a surface. A family $D_{1}, \ldots, D_{r}$ of prime divisors is exceptional if and only if its intersection matrix $\left(D_{i} \cdot D_{j}\right)$ is negative definite.

In this paper, we need the following properties of the modified nef cone $\mathcal{M N}$ and of the divisorial Zariski decomposition, due to Boucksom [Bou04]. We state these properties without proofs.

Theorem 2.11. Let $\alpha \in H^{1,1}(X, \mathbb{R})$ be a pseudo-effective class.

(i) The Zariski projection $Z(\alpha)$ is a modified nef class.

(ii) We have $Z(\alpha)=\alpha$ if and only if $\alpha$ is modified nef.

(iii) The class $Z(\alpha)$ is big if and only if $\alpha$ is.

Remark 2.12. Let $X$ be a complex Kähler surface. For a big class $\alpha \in H^{1,1}(X, \mathbb{R})$, the class $Z(\alpha)$ is a big and modified nef class. By Remark 2.5, any modified nef class is nef; it follows that $Z(\alpha)$ is big and nef.

Theorem 2.13. (i) The map $\alpha \mapsto N(\alpha)$ is convex and homogeneous on the pseudo-effective class cone $\mathcal{E}$. It is continuous on the interior of $\mathcal{E}$.

(ii) The Zariski projection $Z: \mathcal{E} \rightarrow \mathcal{M N}$ is concave and homogeneous. It is continuous on the interior of $\mathcal{E}$.

TheOrem 2.14. Let $p$ be a big and modified nef class. Then the primes $D_{1}, \ldots, D_{q}$ contained in the non-Kähler locus $E_{n K}(p)$ form an exceptional family $A$, and the fiber of $Z$ over $p$ is the simplicial cone $Z^{-1}(p)=p+V_{+}(A)$, where $V_{+}(A):=\sum_{D \in A} \mathbb{R}_{+}\{D\}$. 


\section{Y. DENG}

TheOREM 2.15. Let $X$ be a compact surface. If $\alpha \in H^{1,1}(X, \mathbb{R})$ is a pseudo-effective class, its divisorial Zariski decomposition $\alpha=Z(\alpha)+\{N(\alpha)\}$ is the unique orthogonal decomposition of $\alpha$ with respect to the non-degenerate quadratic form $q(\alpha):=\int \alpha^{2}$ into the sum of a modified nef class and the class of an exceptional effective $\mathbb{R}$-divisor.

Remark 2.16. Let $X$ be a surface; if $\alpha$ is the class of an effective $\mathbb{Q}$-divisor $D$ on a projective surface, then the divisorial Zariski decomposition of $\alpha$ is just the original Zariski decomposition of $D$.

\section{Transcendental Morse inequality}

\subsection{Proof of the transcendental Morse inequality for complex surfaces}

The main goal of this section is to prove the differentiability of the volume function and the transcendental Morse inequality for complex surfaces. In fact, in the next subsection we will give a more general method to prove the transcendental Morse inequality for Kähler manifolds on which modified nef cones $\mathcal{M N}$ coincide with the nef cones $\mathcal{N}$; this includes Kähler surfaces. However, since the methods and results here are very special in studying generalized Okounkov bodies, we will treat complex surface and higher-dimensional Kähler manifolds separately. Throughout this subsection, if not mentioned otherwise, $X$ will stand for a complex Kähler surface. We denote by $q(\alpha):=\int \alpha^{2}$ the quadratic form on $H^{1,1}(X, \mathbb{R})$. By the Hodge index theorem, $\left(H^{1,1}(X, \mathbb{R}), q\right)$ has signature $\left(1, h^{1,1}(X)-1\right)$. The open cone $\left\{\alpha \in H^{1,1}(X, \mathbb{R}) \mid q(\alpha)>0\right\}$ thus has two connected components which are convex cones; we denote by $\mathcal{P}$ the component containing the Kähler cone $\mathcal{K}$.

LEMmA 3.1. Let $X$ be a compact Kähler manifold of dimension $n$. If $\alpha \in H^{1,1}(X, \mathbb{R})$ is a big class and $\beta \in H^{1,1}(X, \mathbb{R})$ is a nef class, then $N(\alpha+t \beta) \leqslant N(\alpha)$ as effective $\mathbb{R}$-divisors for $t \geqslant 0$. Furthermore, when $t$ is small enough, the prime components of $N(\alpha+t \beta)$ will be the same as those of $N(\alpha)$.

Proof. Since $\beta$ is nef, by Theorem 2.13, we have

$$
N(\alpha+t \beta) \leqslant N(\alpha)+t N(\beta)=N(\alpha) .
$$

Since the map $\alpha \mapsto N(\alpha)$ is convex on the pseudo-effective class cone $\mathcal{E}$, it is continuous on the interior of $\mathcal{E}$, and thus the theorem follows.

TheOREM 3.2. If $\alpha \in H^{1,1}(X, \mathbb{R})$ is a big class and $\beta \in H^{1,1}(X, \mathbb{R})$ is a nef class, then

$$
\left.\frac{d}{d t}\right|_{t=0} \operatorname{vol}_{X}(\alpha+t \beta)=2 Z(\alpha) \cdot \beta .
$$

Proof. By Lemma 3.1, there exists an $\epsilon>0$ such that when $0 \leqslant t<\epsilon$, we can write $N(\alpha+t \beta)=$ $\sum_{i=1}^{r} a_{i}(t) N_{i}$, where $0<a_{i}(t) \leqslant a_{i}(0)=: a_{i}$ and each $a_{i}(t)$ is a continuous and decreasing function with respect to $t$. According to the orthogonal property of divisorial Zariski decomposition (Theorem 2.15), we have $Z(\alpha+t \beta) \cdot N(\alpha+t \beta)=0$ for $t \geqslant 0$. Since $Z(\alpha+t \beta)$ is modified nef and thus nef (by Remark 2.6), we have $Z(\alpha+t \beta) \cdot N_{i} \geqslant 0$ for every $i$. When $0 \leqslant t<\epsilon$, we have $a_{i}(t)>0$ for $i=1, \ldots, r$; therefore, $Z(\alpha+t \beta)$ is orthogonal to each $\left\{N_{i}\right\}$ with respect to $q$. We denote by $V \subset H^{1,1}(X, \mathbb{R})$ the finite vector space spanned by $\left\{N_{1}\right\}, \ldots,\left\{N_{r}\right\}$, by $V^{\perp}$ the orthogonal space of $V$ with respect to $q$. Thus $\alpha+t \beta=Z(\alpha+t \beta)+\sum_{i=1}^{r} a_{i}(t)\left\{N_{i}\right\}$ is the decomposition in the direct sum $V^{\perp} \oplus V$. We decompose $\beta=\beta^{\perp}+\beta_{0}$ in the direct sum $V^{\perp} \oplus V$; 


\section{Generalized Okounkov Bodies}

we have

$$
\begin{aligned}
Z(\alpha+t \beta) & =Z(\alpha)+t \beta^{\perp}, \\
\sum_{i=1}^{r} a_{i}(t)\left\{N_{i}\right\} & =\sum_{i=1}^{r} a_{i}\left\{N_{i}\right\}+t \beta_{0} .
\end{aligned}
$$

Since $\operatorname{vol}_{X}(\alpha+t \beta)=\operatorname{vol}_{X}(Z(\alpha+t \beta))=Z(\alpha+t \beta)^{2}$ (by Remark 2.8), it is easy to deduce that

$$
\left.\frac{d}{d t}\right|_{t=0} \operatorname{vol}_{X}(\alpha+t \beta)=2 Z(\alpha) \cdot \beta^{\perp}=2 Z(\alpha) \cdot \beta .
$$

The last equality follows from the inclusions $\beta_{0} \in V$ and $Z(\alpha) \in V^{\perp}$. We have proved the first half of Theorem 1.5.

To prove the transcendental Morse inequality for complex surfaces, we will need a criterion for the bigness of a class.

Theorem 3.3. Let $\alpha$ and $\beta$ be two nef classes such that $\alpha^{2}-2 \alpha \cdot \beta>0$; then $\alpha-\beta$ is a big class.

Proof. Recall that $\mathcal{P}$ is the connected component of the open cone $\left\{\alpha \in H^{1,1}(X, \mathbb{R}) \mid q(\alpha)>0\right\}$ containing the Kähler cone $\mathcal{K}$; then $\mathcal{P} \subset \mathcal{E}^{0}$. As a consequence of the Nakai-Moishezon criterion for surfaces [Lam99], we know that if $\gamma$ is a real $(1,1)$-class with $\gamma^{2}>0$, then $\gamma$ or $-\gamma$ is big. Since $\alpha$ and $\beta$ are both nef, we have $(\alpha-t \beta)^{2}>0$ for $0 \leqslant t \leqslant 1$. This means that $\alpha-t \beta$ is contained in some component of the open cone $\left\{\alpha \in H^{1,1}(X, \mathbb{R}) \mid q(\alpha)>0\right\}$. But since $\alpha$ is big, $\alpha-t \beta$ is contained in $\mathcal{P} \subset \mathcal{B}$, and a fortiori $\alpha-\beta$ is.

Now, we are ready to prove the transcendental Morse inequality for complex surfaces.

Proof of Theorem 1.6. By Theorem 3.3, when $\alpha^{2}-2 \alpha \cdot \beta>0$, the cohomology class $\alpha-\beta$ is big. By the differentiability formula (3.1), we have

$$
\operatorname{vol}_{X}(\alpha-\beta)=\alpha^{2}-2 \int_{0}^{1} Z(\alpha-t \beta) \cdot \beta d t
$$

Since the Zariski projection $Z: \mathcal{E} \rightarrow \mathcal{M N}$ is concave and homogeneous by Theorem 2.13, we have

$$
\alpha=Z(\alpha) \geqslant Z(\alpha-t \beta)+Z(t \beta) \geqslant Z(\alpha-t \beta) .
$$

Since $\beta$ is nef, we have

$$
\alpha \cdot \beta \geqslant Z(\alpha-t \beta) \cdot \beta
$$

and thus

$$
\operatorname{vol}_{X}(\alpha-\beta) \geqslant \alpha^{2}-2 \alpha \cdot \beta
$$

In the last part of this subsection, we prove the second half of Theorem 1.5.

Theorem 3.4. Let $\alpha \in H^{1,1}(X, \mathbb{R})$ be a big class, and let $C$ be an irreducible divisor; then

$$
\left.\frac{d}{d t}\right|_{t=0} \operatorname{vol}_{X}(\alpha+t C)=2 Z(\alpha) \cdot C .
$$




\section{Y. DENG}

Proof. It suffices to prove the theorem for $C$ not nef. Thus we have $C^{2}<0$. Write $N(\alpha)=$ $\sum_{i=1}^{r} a_{i} N_{i}$, where each $N_{i}$ is prime divisor. If $C \subseteq E_{n K}(Z(\alpha))$, we deduce that $Z(\alpha) \cdot C=0$ by Theorem 2.2, and $\left\{C, N_{1}, \ldots, N_{r}\right\}$ forms an exceptional family by Theorem 2.14. Thus we have

$$
Z(\alpha+t C)=Z(\alpha) \text { and } \quad N(\alpha+t C)=N(\alpha)+t C
$$

for $t \geqslant 0$. The theorem is thus proved in this case.

From now on we assume $C \nsubseteq E_{n K}(Z(\alpha))$; thus we have $Z(\alpha) \cdot C>0$ and $C \nsubseteq \operatorname{Supp}(N(\alpha))$. We define

$$
\left(\begin{array}{c}
b_{1} \\
\vdots \\
b_{r}
\end{array}\right)=-S^{-1} \cdot\left(\begin{array}{c}
C \cdot N_{1} \\
\vdots \\
C \cdot N_{r}
\end{array}\right),
$$

where $S=\left(s_{i j}\right)$ denotes the intersection matrix of $\left\{N_{1}, \ldots, N_{r}\right\}$. By Theorem 2.15 we know that $S$ is negative definite, satisfying $s_{i j} \geqslant 0$ for all $i \neq j$. We claim that $Z(\alpha)+t\left(\{C\}+\sum_{i=1}^{r} b_{i}\left\{N_{i}\right\}\right)$ is big and nef if $0 \leqslant t<-(Z(\alpha) \cdot C) / C^{2}$. We need the following lemma from [BKS04] to prove our claim.

Lemma 3.5. Let $A$ be a negative-definite $r \times r$-matrix over the reals such that $a_{i j} \geqslant 0$ for all $i \neq j$. Then all entries of the inverse matrix $A^{-1}$ are at most 0 .

By Lemma 3.5, we know that all entries of $S^{-1}$ are at most 0 ; thus $b_{j} \geqslant 0$ for all $1 \leqslant j \leqslant r$ and we get the bigness of $Z(\alpha)+t\left(\{C\}+\sum_{i=1}^{r} b_{i}\left\{N_{i}\right\}\right)$. By the construction of $b_{j}$, we have

$$
\left(Z(\alpha)+t\left(\{C\}+\sum_{i=1}^{r} b_{i}\left\{N_{i}\right\}\right)\right) \cdot N_{j}=0
$$

for $1 \leqslant j \leqslant r$ and

$$
\left(Z(\alpha)+t\left(\{C\}+\sum_{i=1}^{r} b_{i}\left\{N_{i}\right\}\right)\right) \cdot C>0
$$

for $0 \leqslant t<-(Z(\alpha) \cdot C) / C^{2}$. Thus we have the nefness and our claim follows. Since the divisorial Zariski decomposition is orthogonal and unique (see Theorem 2.15), we conclude that

$$
\begin{aligned}
& N(\alpha+t\{C\})=\sum_{i=1}^{r}\left(a_{i}-t b_{i}\right) N_{i} \\
& Z(\alpha+t\{C\})=Z(\alpha)+t\{C\}+\sum_{i=1}^{r} t b_{i}\left\{N_{i}\right\}
\end{aligned}
$$

for $t$ small enough. Since $\operatorname{vol}_{X}(\alpha+t C)=Z(\alpha+t\{C\})^{2}$, we have thus also obtained formula (3.2) in this case.

\subsection{Transcendental Morse inequality for some special Kähler manifolds}

One can modify the proof of Theorem 1.6 a little bit, to extend the transcendental Morse inequality to Kähler manifolds whose modified nef cone $\mathcal{M N}$ coincides with the nef cone $\mathcal{N}$. In this subsection, we assume $X$ to be a compact Kähler manifold of dimension $n$ which satisfies this condition.

Lemma 3.6. If $\alpha \in \mathcal{E}^{\circ}$, then the divisorial Zariski decomposition $\alpha=Z(\alpha)+N(\alpha)$ is such that

$$
Z(\alpha)^{n-1} \cdot N(\alpha)=0 .
$$




\section{Generalized Okounkov Bodies}

Remark 3.7. Lemma 3.6 is very similar to the Corollary 4.5 in [BDPP13]: if $\alpha \in \mathcal{E}_{\mathrm{NS}}$, then the divisorial Zariski decomposition $\alpha=Z(\alpha)+N(\alpha)$ is such that $\left\langle Z(\alpha)^{n-1}\right\rangle \cdot N(\alpha)=0$. However, the proof of [BDPP13] is based on the orthogonal estimate for the divisorial Zariski decomposition of $\mathcal{E}_{\mathrm{NS}}$, which is still a conjecture for $\alpha \in \mathcal{E}$. Here we will use Theorem 2.2 to prove this lemma directly.

Proof of Lemma 3.6. By Theorem 2.11, if $\alpha$ is big, then $Z(\alpha)$ is big and modified nef, thus nef by the assumption for $X$. By Theorem 2.14, the primes $D_{1}, \ldots, D_{q}$ contained in the nonKähler locus $E_{n K}(Z(\alpha))$ form an exceptional family, and $\alpha=Z(\alpha)+\sum_{i=1}^{r} a_{i} D_{i}$ for $a_{i} \geqslant 0$. Since $\operatorname{Null}(Z(\alpha))=E_{n K}(Z(\alpha))$ by Theorem 2.2, we have $Z(\alpha)^{n-1} \cdot D_{i}=0$ for each $i$, and thus $Z(\alpha)^{n-1} \cdot N(\alpha)=0$. The lemma is proved.

Proof of Theorem 1.7. By Lemma 3.1, there exists an $\epsilon>0$ such that the prime components of $N(\alpha+t \beta)$ will be the same as those of $N(\alpha)$ when $0 \leqslant t \leqslant \epsilon$. Moreover, if we write $N(\alpha+t \beta)=$ $\sum_{i=1}^{r} a_{i}(t) N_{i}$, then each $a_{i}(t)$ is continuous and decreasing, satisfying $a_{i}(t)>0$. By Lemma 3.6, we have

$$
Z(\alpha+t \beta)^{n-1} \cdot N(\alpha+t \beta)=\sum_{i=1}^{r} a_{i}(t) Z(\alpha+t \beta)^{n-1} \cdot N_{i}=0 .
$$

Since $Z(\alpha+t \beta)$ is modified nef, thus nef, we deduce that $Z(\alpha+t \beta)^{n-1} \cdot N_{i}=0$ for $0 \leqslant t \leqslant \epsilon$ and $i=1, \ldots, r$.

Since $a_{i}(t)$ is continuous and decreasing, it is almost everywhere differentiable. Thus $Z(\alpha+$ $t \beta)=\alpha+t \beta-\sum_{i=1}^{r} a_{i}(t) N_{i}$ is an a.e. differentiable and continuous curve in the finite-dimensional space $H^{1,1}(X, \mathbb{R})$ parametrized by $t$. Meanwhile, since $\alpha \mapsto \alpha^{n}$ is a polynomial in $H^{1,1}(X, \mathbb{R})$, we thus deduce that $\operatorname{vol}_{X}(\alpha+t \beta)=Z(\alpha+t \beta)^{n}$ is an a.e. differentiable function with respect to $t$. Therefore, if $\operatorname{vol}_{X}(\alpha+t \beta)$ and $a_{i}(t)$ are both differentiable at $t=t_{0}$, we have

$$
\left.\frac{d}{d t}\right|_{t=t_{0}} \operatorname{vol}_{X}(\alpha+t \beta)=n Z\left(\alpha+t_{0} \beta\right)^{n-1} \cdot\left(\beta-\sum_{i=1}^{r} a_{i}{ }^{\prime}\left(t_{0}\right) N_{i}\right)=n Z\left(\alpha+t_{0} \beta\right)^{n-1} \cdot \beta .
$$

Since $\operatorname{vol}_{X}(\alpha+t \beta)$ is increasing and continuous, it is also a.e. differentiable, and thus we have

$$
\begin{aligned}
\operatorname{vol}_{X}(\alpha+s \beta) & =\operatorname{vol}_{X}(\alpha)+\int_{0}^{s} \frac{d}{d t} \operatorname{vol}_{X}(\alpha+t \beta) \mathrm{d} t \\
& =\operatorname{vol}_{X}(\alpha)+n \int_{0}^{s} Z(\alpha+t \beta)^{n-1} \cdot \beta \mathrm{d} t
\end{aligned}
$$

for $0 \leqslant s \leqslant \epsilon$. Since $Z(\alpha+t \beta)$ is continuous (by Theorem 2.13), by (3.3) we deduce that $\operatorname{vol}_{X}(\alpha+t \beta)$ is differentiable with respect to $t$. Its derivative is

$$
\left.\frac{d}{d t}\right|_{t=t_{0}} \operatorname{vol}_{X}(\alpha+t \beta)=n Z\left(\alpha+t_{0} \beta\right)^{n-1} \cdot \beta .
$$

To prove the transcendental Morse inequality, we will need the following bigness criterion obtained in [Xia13] and [Pop16].

TheOrem 3.8. Let $X$ be an $n$-dimensional compact Kähler manifold. Assume that $\alpha$ and $\beta$ are two nef classes on $X$ satisfying $\alpha^{n}-n \alpha^{n-1} \cdot \beta>0$; then $\alpha-\beta$ is a big class.

The proof of the next theorem is similar to that of Theorem 1.6 and is therefore omitted.

Theorem 3.9. Let $X$ be a compact Kähler manifold on which the modified nef cone $\mathcal{M N}$ and the nef cone $\mathcal{N}$ coincide. If $\alpha$ and $\beta$ are nef cohomology classes of type $(1,1)$ on $X$ satisfying 


\section{Y. DENG}

the inequality $\alpha^{n}-n \alpha^{n-1} \cdot \beta>0$, then $\alpha-\beta$ contains a Kähler current and $\operatorname{vol}_{X}(\alpha-\beta) \geqslant$ $\alpha^{n}-n \alpha^{n-1} \cdot \beta$.

Remark 3.10. In [BFJ09], the authors proved the following differentiability theorem:

$$
\left.\frac{d}{d t}\right|_{t=t_{0}} \operatorname{vol}_{X}(L+t D)=n\left\langle L^{n-1}\right\rangle \cdot D,
$$

where $L$ is a big line bundle on the smooth projective variety $X$ and $D$ is a prime divisor. The right-hand side of (3.4) involves the positive intersection product $\left\langle L^{n-1}\right\rangle \in H_{\geqslant 0}^{n-1, n-1}(X, \mathbb{R})$, first introduced in the analytic context in [BDPP13]. Theorem 1.7 could be seen as a transcendental version of (3.4) for some special Kähler manifolds. In the general Kähler situation, we propose the following conjecture.

Conjecture 3.11. Let $X$ be a Kähler manifold of dimensional $n$, and let $\alpha$ be a big class. If $\beta$ is a pseudo-effective class, then we have

$$
\left.\frac{d}{d t}\right|_{t=0} \operatorname{vol}_{X}(\alpha+t \beta)=n\left\langle\alpha^{n-1}\right\rangle \cdot \beta .
$$

\section{Generalized Okounkov bodies on Kähler manifolds}

\subsection{Definition and relation with the algebraic case}

Throughout this subsection, $X$ will stand for a Kähler manifold of dimension $n$. Our main goal in this subsection is to generalize the definition of Okounkov body to any pseudo-effective class $\alpha \in H^{1,1}(X, \mathbb{R})$. First of all, we define a valuation-like function. For any positive current $T \in \alpha$ with analytic singularities, we define the valuation-like function

$$
T \mapsto \nu(T)=\nu_{Y_{\bullet}}(T)=\left(\nu_{1}(T), \ldots, \nu_{n}(T)\right)
$$

as follows. First, set

$$
\nu_{1}(T)=\sup \left\{\lambda \mid T-\lambda\left[Y_{1}\right] \geqslant 0\right\},
$$

where $\left[Y_{1}\right]$ is the current of integration over $Y_{1}$. By Section 2.1, we know that $\nu_{1}(T)$ is the coefficient $\nu\left(T, Y_{1}\right)$ of the positive current $\left[Y_{1}\right]$ appearing in the Siu decomposition of $T$. Since $T$ has analytic singularities, by the arguments in Section 2.2, the current $T_{1}:=\left.\left(T-\nu_{1}\left[Y_{1}\right]\right)\right|_{Y_{1}}$ is a well-defined positive current in the pseudo-effective class $\left.\left(\alpha-\nu_{1}\left\{Y_{1}\right\}\right)\right|_{Y_{1}}$, and it also has analytic singularities. Then take

$$
\nu_{2}(T)=\sup \left\{\lambda \mid T_{1}-\lambda\left[Y_{2}\right] \geqslant 0\right\}
$$

and continue in this manner to define the remaining values $\nu_{i}(T) \in \mathbb{R}^{+}$.

Remark 4.1. If one assumes $\alpha \in \mathrm{NS}_{\mathbb{Z}}(X)$, there exists a holomorphic line bundle $L$ such that $\alpha=c_{1}(L)$. If $D$ is the divisor of some holomorphic section $s_{D} \in H^{0}\left(X, \mathcal{O}_{X}(L)\right)$, then we have

$$
\nu([D])=\mu\left(s_{D}\right),
$$

where $\mu$ is the valuation-like function appearing in the definition of the original Okounkov body. Roughly speaking, our definition of the valuation-like function has a bigger domain of definition and thus the image of our valuation-like function contains $\bigcup_{m=1}^{\infty} \frac{1}{m} \mu(m L)$.

For any big class $\alpha$, we define the $\mathbb{Q}$-convex body $\Delta_{\mathbb{Q}}(\alpha)$ (respectively, $\mathbb{R}$-convex body $\Delta_{\mathbb{R}}(\alpha)$ ) to be the set of valuation vectors $\nu(T)$, where $T$ ranges over all the Kähler (respectively, positive) currents with algebraic (respectively, analytic) singularities. Then $\Delta_{\mathbb{Q}}(\alpha) \subseteq \Delta_{\mathbb{R}}(\alpha)$. It is easy to 


\section{Generalized Okounkov Bodies}

check that this is a convex set in $\mathbb{Q}^{n}$ (respectively, $\mathbb{R}^{n}$ ). Indeed, for any two positive currents $T_{0}$ and $T_{1}$ with algebraic (respectively, analytic) singularities, we have $\nu\left(\epsilon T_{0}+(1-\epsilon) T_{1}\right)=$ $\epsilon \nu\left(T_{0}\right)+(1-\epsilon) \nu\left(T_{1}\right)$ for $0 \leqslant \epsilon \leqslant 1$ rational (respectively, real). Also obvious, is the homogeneous property of $\Delta_{\mathbb{Q}}(\alpha)$; that is, for all $c \in \mathbb{Q}^{+}$, we have

$$
\Delta_{\mathbb{Q}}(c \alpha)=c \Delta_{\mathbb{Q}}(\alpha) .
$$

Indeed, since we have $\nu(c T)=c \nu(T)$ for all $c \in \mathbb{R}^{+}$, the claim follows directly.

EXAmPLE 4.2. Let $L$ be a line bundle of degree $c>0$ on a smooth curve $C$ of genus $g$. Then we have

$$
\Delta_{\mathbb{Q}}\left(c_{1}(L)\right)=\mathbb{Q} \cap[0, c) .
$$

Since $\mathrm{NS}_{\mathbb{R}}(C)=H^{1,1}(C, \mathbb{R})$, for any ample class $\alpha$ on $C$, we have

$$
\Delta_{\mathbb{Q}}(\alpha)=\mathbb{Q} \cap[0, \alpha \cdot C) .
$$

Lemma 4.3. Let $\alpha$ be a big class; then the $\mathbb{R}$-convex body $\Delta_{\mathbb{R}}(\alpha)$ lies in a bounded subset of $\mathbb{R}^{n}$.

Proof. It suffices to show that there exists a $b>0$ large enough that $\nu_{i}(T)<b$ for any positive current $T$ with analytic singularities. We fix a Kähler class $\omega$. First of all, choose $b_{1}>0$ such that

$$
\left(\alpha-b_{1} Y_{1}\right) \cdot \omega^{n-1}<0 .
$$

This guarantees that $\nu_{1}(T)<b_{1}$ since $\alpha-b_{1} Y_{1} \notin \mathcal{E}$. Next choose $b_{2}$ large enough that

$$
\left(\left.\left(\alpha-a Y_{1}\right)\right|_{Y_{1}}-b_{2} Y_{2}\right) \cdot \omega^{n-2}<0
$$

for all real numbers $0 \leqslant a \leqslant b_{1}$. Then $\nu_{2}(T) \leqslant b_{2}$ for any positive current $T$ with analytic singularities. Continuing in this manner, we construct $b_{i}>0$ for $i=1, \ldots, n$ such that $\nu_{i}(T) \leqslant b_{i}$ for any positive current $T$ with analytic singularities. We take $b=\max \left\{b_{i}\right\}$.

Lemma 4.4. For any big class $\alpha$, the $\mathbb{Q}$-convex body $\Delta_{\mathbb{Q}}(\alpha)$ is dense in $\Delta_{\mathbb{R}}(\alpha)$; in particular, we have $\overline{\Delta_{\mathbb{Q}}(\alpha)}=\overline{\Delta_{\mathbb{R}}(\alpha)}$.

Proof. It is easy to verify that if $T$ is a Kähler current with analytic singularities, then for any $\epsilon>0$, there exists a Kähler current $S_{\epsilon}$ with algebraic singularities such that $\left\|\nu\left(S_{\epsilon}\right)-\nu(T)\right\|<\epsilon$ with respect to the standard norm in $\mathbb{R}^{n}$. For the general case, we fix a Kähler current $T_{0} \in$ $i \Theta(L)$ with algebraic singularities. Then for any positive current $T$ with analytic singularities, $T_{\epsilon}:=(1-\epsilon) T+\epsilon T_{0}$ is still a Kähler current. By Lemma 4.3, the norm $\left\|\nu\left(T_{\epsilon}\right)-\nu(T)\right\|=$ $\epsilon\left\|\left(\nu\left(T_{0}\right)-\nu(T)\right)\right\|$ will tend to 0 since $\nu(T)$ is uniformly bounded for any positive current $T$ with analytic singularities. Thus $\Delta_{\mathbb{Q}}(\alpha)$ is dense in $\Delta_{\mathbb{R}}(\alpha)$.

Now, we study the relations between $\Delta_{\mathbb{Q}}\left(c_{1}(L)\right)$ and $\Delta(L)$ for $L$ a big line bundle on $X$. We begin with the following two lemmas.

Lemma 4.5. Let $L$ be a big line bundle on the projective variety $X$ of dimension $n$, with a singular Hermitian metric $h=e^{-\varphi}$ satisfying

$$
i \Theta_{L, h}=d d^{c} \varphi \geqslant \epsilon \omega
$$

for some $\epsilon>0$ and a given Kähler form $\omega$. If the restriction of $\varphi$ on a smooth hypersurface $Y$ is not identically equal to $-\infty$, then there exists a positive integer $m_{0}$ which depends only on $Y$ such that any holomorphic section $s_{m} \in H^{0}\left(Y, \mathcal{O}_{Y}(m L) \otimes \mathcal{I}\left(\left.m \varphi\right|_{Y}\right)\right)$ can be extended to $S_{m} \in H^{0}\left(X, \mathcal{O}_{X}(m L) \otimes \mathcal{I}(m \varphi)\right)$ for any $m \geqslant m_{0}$. 


\section{Y. Deng}

We need the following Ohsawa-Takegoshi extension theorem to prove Lemma 4.5.

TheOREM 4.6. Let $X$ be a smooth projective variety, and let $Y$ be a smooth divisor defined by a holomorphic section of the line bundle $H$ with a smooth metric $h_{0}=e^{-\psi}$. If $L$ is a holomorphic line bundle with a singular metric $h=e^{-\phi}$, satisfying the curvature assumptions

$$
d d^{c} \phi \geqslant 0 \text { and } d d^{c} \phi \geqslant \delta d d^{c} \psi
$$

with $\delta>0$, then for any holomorphic section $s \in H^{0}\left(Y, \mathcal{O}_{Y}\left(K_{Y}+L\right) \otimes \mathcal{I}\left(\left.h\right|_{Y}\right)\right)$, there exists a global holomorphic section $S \in H^{0}\left(X, \mathcal{O}_{X}\left(K_{X}+L+Y\right) \otimes \mathcal{I}(h)\right)$ such that $\left.S\right|_{Y}=s$.

Proof of Lemma 4.5. Taking smooth metrics $e^{-\psi}$ and $e^{-\eta}$ on $Y$ and $K_{X}$, respectively, we can choose $m_{0}$ large enough for the curvature assumptions

$$
d d^{c}(m \phi-\eta-\psi) \geqslant 0
$$

and

$$
d d^{c}(m \phi-\eta-\psi) \geqslant d d^{c} \psi
$$

to hold for any $m \geqslant m_{0}$.

By Theorem 4.6, any holomorphic section $s \in H^{0}\left(Y, \mathcal{O}_{Y}\left(K_{Y}+\left.\left(m L-K_{X}-Y\right)\right|_{Y}\right) \otimes \mathcal{I}\left(\left.h^{m}\right|_{Y}\right)\right)$ can be extended to a global holomorphic section $S \in H^{0}\left(X, \mathcal{O}_{X}(m L) \otimes \mathcal{I}\left(h^{m}\right)\right)$ such that $\left.S\right|_{Y}=s$. By the adjunction theorem, we have $\left.\left(K_{X}+Y\right)\right|_{Y}=K_{Y}$; thus the lemma is proved.

Lemma 4.7. Let $L$ be a big line bundle on the Riemann surface $C$ with a singular Hermitian metric $h=e^{-\varphi}$ such that $\varphi$ has algebraic singularities and

$$
i \Theta_{L, h}=d d^{c} \varphi \geqslant \epsilon \omega
$$

for some $\epsilon>0$. Then for a fixed point $p$, there exists an integer $k>0$ such that we have a holomorphic section $s_{k} \in H^{0}\left(C, \mathcal{O}_{C}(k L) \otimes \mathcal{I}\left(h^{k}\right)\right)$ satisfying $\operatorname{ord}_{p}\left(s_{k}\right)=k \nu\left(i \Theta_{L, h}, p\right)$.

Proof. Since $\varphi$ has algebraic singularities, we have the following Lebesgue decomposition:

$$
i \Theta_{L, h}=\left(i \Theta_{L, h}\right)_{\mathrm{ac}}+\sum_{i=1}^{r} c_{i} x_{i},
$$

where each $c_{i}>0$ is rational and $x_{1}, \ldots, x_{r}$ are the log poles of $i \Theta_{L, h}$ (possibly $p$ is among them). We have

$$
\int_{C} i\left(\Theta_{L, h}\right)_{\mathrm{ac}}+\sum_{i=1}^{r} c_{i}=\operatorname{deg}(L)
$$

thus

$$
\sum_{i=1}^{r} c_{i}<\operatorname{deg}(L) .
$$

By the Riemann-Roch theorem, there exists an integer $k>0$ such that

(i) $k c_{i}$ is an integer,

(ii) there is a holomorphic section $s_{k} \in H^{0}\left(C, \mathcal{O}_{C}(k L)\right)$ such that $\operatorname{ord}_{x_{i}}\left(s_{k}\right) \geqslant k c_{i}$ and $\operatorname{ord}_{p}\left(s_{k}\right)$ $=k \nu\left(i \Theta_{L, h}, p\right)$.

Thus $s_{k}$ is locally integrable with respect to the weight $e^{-k \varphi}$. The theorem is proved. 


\section{Generalized Okounkov Bodies}

Theorem 4.8. Let $X$ be a smooth projective manifold of dimension $n$. For any Kähler current $T \in c_{1}(L)$ with algebraic singularities, there exists a holomorphic section $s \in H^{0}\left(X, \mathcal{O}_{X}(k L)\right)$ such that $\mu(s)=k \nu(T)$; that is, we have

$$
\nu(T) \in \bigcup_{m=1}^{\infty} \frac{1}{m} \mu(m L) .
$$

In particular,

$$
\Delta_{\mathbb{Q}}\left(c_{1}(L)\right) \subseteq \bigcup_{m=1}^{\infty} \frac{1}{m} \mu(m L) \subseteq \Delta(L) .
$$

Proof. First, set $\nu_{i}=\nu_{i}(T)$ and define

$$
\begin{aligned}
T_{0} & :=T, T_{1}:=\left.\left(T_{0}-\nu_{1}\left[Y_{1}\right]\right)\right|_{Y_{1}}, \ldots, T_{n-1}:=\left.\left(T_{n-2}-\nu_{n-1}\left[Y_{n-1}\right]\right)\right|_{Y_{n-1}} ; \\
L_{0} & :=L-\nu_{1} Y_{1}, L_{1}:=\left.L_{0}\right|_{Y_{1}}-\nu_{2} Y_{2}, \ldots, L_{n-2}:=\left.L_{n-3}\right|_{Y_{n-2}}-\nu_{n-1} Y_{n-1} .
\end{aligned}
$$

Since $T_{0} \geqslant \epsilon \omega$, we have $T_{1} \geqslant\left.\epsilon \omega\right|_{Y_{1}}, \ldots, T_{n-1} \geqslant\left.\epsilon \omega\right|_{Y_{n-1}}$. Since each $\nu_{i}$ is rational, we can find an integer $m$ such that each $m \nu_{i}$ is an integer, so that each $m L_{i}$ is a big line bundle on $Y_{i}$. If we can prove

$$
\nu(m T) \in \bigcup_{k=1}^{\infty} \frac{1}{k} \mu(k m L)
$$

then we will have

$$
\nu(T) \in \bigcup_{m=1}^{\infty} \frac{1}{m} \mu(m L),
$$

by the homogeneous property $\frac{1}{m} \nu(m T)=\nu(T)$. Thus we can assume that each $\nu_{i}(T)$ is an integer after we replace $L$ by $m L$ and $T$ by $m T$.

First, since $T_{0} \in c_{1}(L)$ is a Kähler current with algebraic singularities, there exists a singular metric $h=e^{-\varphi_{0}}$ on $L$ whose curvature current is $T_{0}$ and such that $\varphi$ has algebraic singularities; on the other hand, there is a canonical metric $e^{-\eta_{0}}$ on $\mathcal{O}_{Y_{0}}\left(Y_{1}\right)$ such that $d d^{c} \eta_{0}=\left[Y_{1}\right]$ in the sense of currents. Thus by the definition of $\nu_{1}$, we deduce that $h_{0}:=e^{-\varphi_{0}+\nu_{1} \eta_{0}}$ is a singular metric of $L_{0}$ such that $-\varphi_{0}+\nu_{1} \eta_{0}$ does not vanish identically on $Y_{1}$ and $\left.h_{0}\right|_{Y_{1}}$ is a singular metric of $\left.L_{0}\right|_{Y_{1}}$ with algebraic singularities whose curvature current is $T_{1} \geqslant\left.\epsilon \omega\right|_{Y_{1}}$.

Second, there is a canonical singular metric $e^{-\eta_{1}}$ of $\mathcal{O}_{Y_{1}}\left(Y_{2}\right)$ on $Y_{1}$ with curvature current $\left[Y_{2}\right]$. Thus, the singular metric $h_{1}:=\left.h_{0}\right|_{Y_{1}}+e^{\nu_{2} \eta_{1}}$ of the big line bundle $L_{1}$ gives a curvature current $T_{1}-\nu_{2}\left[Y_{2}\right] \geqslant\left.\epsilon \omega\right|_{Y_{1}}$. We continue in this manner to define the remaining singular metrics $h_{i}:=$ $\left.h_{i-1}\right|_{Y_{i}}+e^{\nu_{i+1} \eta_{i}}$ of the big line bundle $L_{i}$ on $Y_{i}$ with curvature current $T_{i}-\nu_{i+1}\left[Y_{i+1}\right] \geqslant\left.\epsilon \omega\right|_{Y_{i}}$ for $i=0, \ldots, n-1$. It is easy to see that $\left.h_{i}\right|_{Y_{i+1}}$ is well defined.

By Lemma 4.5, there exists a $k_{0}$ such that for each $k \geqslant k_{0}$, the following short sequence is exact for $i=1, \ldots, n-1$ :

$$
H^{0}\left(Y_{i-1}, \mathcal{O}_{Y_{i-1}}\left(k L_{i-1}\right) \otimes \mathcal{I}\left(h_{i-1}^{k}\right)\right) \longrightarrow H^{0}\left(Y_{i}, \mathcal{O}_{Y_{i}}\left(k L_{i-1}\right) \otimes \mathcal{I}\left(\left.h_{i-1}^{k}\right|_{Y_{i}}\right)\right) \longrightarrow 0
$$

Now, we begin our construction. The current $T_{n-1}$ is the curvature current of the singular metric $\left.h_{n-2}\right|_{Y_{n-1}}$ of $\left.L_{n-2}\right|_{Y_{n-1}}$ over the Riemann surface $Y_{n-1}$. By Lemma 4.7, there exist a $k \geqslant k_{0}$ and a holomorphic section $s_{n-1} \in H^{0}\left(Y_{n-1}, \mathcal{O}_{Y_{n-1}}\left(k L_{n-2}\right) \otimes \mathcal{I}\left(\left.h_{n-2}^{k}\right|_{Y_{n-1}}\right)\right)$, such that $\operatorname{ord}_{p}\left(s_{n-1}\right)=k \nu\left(T_{n-1}, p\right)=k \nu_{n}$. 


\section{Y. DENG}

By the exact sequence (4.1), $s_{n-1}$ can be extend to

$$
\widetilde{s}_{n-2} \in H^{0}\left(Y_{n-2}, \mathcal{O}_{Y_{n-2}}\left(k L_{n-2}\right) \otimes \mathcal{I}\left(h_{n-2}^{k}\right)\right) .
$$

Now, we choose a canonical section $t_{n-2}$ of $H^{0}\left(Y_{n-2}, \mathcal{O}_{Y_{n-2}}\left(Y_{n-1}\right)\right)$ such that the divisor of $t_{n-2}$ is $Y_{n-1}$. We define $s_{n-2}:=\widetilde{s}_{n-2} t_{n-2}^{\otimes \nu_{n-1}}$. By the construction of $h_{n-2}:=\left.h_{n-3}\right|_{Y_{n-2}}+e^{\nu_{n-1} \eta_{n-2}}$, we obtain that

$$
s_{n-2} \in H^{0}\left(Y_{n-2}, \mathcal{O}_{Y_{n-2}}\left(k L_{n-3}\right) \otimes \mathcal{I}\left(\left.h_{n-3}^{k}\right|_{Y_{n-2}}\right)\right) .
$$

We can continue in this manner to construct a section $s_{0} \in H^{0}\left(X, \mathcal{O}_{X}(k L)\right)$, and by our construction we have

$$
\mu\left(s_{0}\right)=\left(k \nu_{1}, \ldots, k \nu_{n}\right)=k \nu(T) .
$$

This concludes the proof of the theorem.

Proposition 4.9. For any big line bundle $L$ and any admissible flag $Y_{\bullet}$, one has $\overline{\Delta_{\mathbb{Q}}\left(c_{1}(L)\right)}=$ $\Delta(L)$. In particular,

$$
\Delta(L)=\overline{\bigcup_{m=1}^{\infty} \frac{1}{m} \nu(m L)} .
$$

Proof. First, since $\Delta_{\mathbb{Q}}\left(c_{1}(L)\right)$ is a convex set in $\mathbb{Q}^{n}$, its closure in $\mathbb{R}^{n}$, denoted by $\overline{\Delta_{\mathbb{Q}}\left(c_{1}(L)\right)}$, is also a closed convex set. By Proposition 4.8, we have

$$
\Delta_{\mathbb{Q}}\left(c_{1}(L)\right) \subset \bigcup_{m=1}^{\infty} \frac{1}{m} \cdot \nu(m L) ;
$$

thus

$$
\overline{\Delta_{\mathbb{Q}}\left(c_{1}(L)\right)} \subseteq \Delta(L) .
$$

By Remark 4.1, we have $\bigcup_{m=1}^{\infty} \frac{1}{m} \nu(m L) \subseteq \Delta_{\mathbb{R}}\left(c_{1}(L)\right)$, thus by the definition of the Okounkov body $\Delta(L)$, we deduce that

$$
\Delta(L) \subseteq \overline{\Delta_{\mathbb{R}}\left(c_{1}(L)\right)} .
$$

By Lemma 4.4, we have $\overline{\Delta_{\mathbb{Q}}\left(c_{1}(L)\right)}=\overline{\Delta_{\mathbb{R}}\left(c_{1}(L)\right)}$; thus the theorem is proved.

Remark 4.10. By Proposition 4.9, in the definition of the Okounkov body $\Delta(L)$, it suffices to close up the set of normalized valuation vectors instead of taking the closure of the convex hull of this set.

Remark 4.11. It is easy to re-prove that the Okounkov body $\Delta(L)$ depends only on the numerical equivalence class of the big line bundle $L$. Indeed, if $L_{1}$ and $L_{2}$ are numerically equivalent, we have $c_{1}\left(L_{1}\right)=c_{1}\left(L_{2}\right)$; thus

$$
\Delta_{\mathbb{Q}}\left(c_{1}\left(L_{1}\right)\right)=\Delta_{\mathbb{Q}}\left(c_{1}\left(L_{2}\right)\right) .
$$

By Proposition 4.9, we have $\Delta\left(L_{1}\right)=\Delta\left(L_{2}\right)$.

Now, we are ready to find some valuative points in the Okounkov bodies.

Proof of Corollary 1.2. From [LM09] we know that $\operatorname{vol}_{\mathbb{R}^{n}}(\Delta(L))=\operatorname{vol}_{X}(L)>0$ by the bigness of $L$. Since we have $\Delta(L)=\overline{\Delta_{\mathbb{Q}}\left(c_{1}(L)\right)}$ by Proposition 4.9 , for any $p \in \operatorname{int}(\Delta(L)) \cap \mathbb{Q}^{n}$, there exists an $n$-simplex $\Delta_{n}$ containing $p$ with all the vertices lying in $\Delta_{\mathbb{Q}}\left(c_{1}(L)\right)$. Since $\Delta_{\mathbb{Q}}\left(c_{1}(L)\right)$ is a convex set in $\mathbb{Q}^{n}$, we have $\Delta_{n} \cap \mathbb{Q}^{n} \subseteq \Delta_{\mathbb{Q}}\left(c_{1}(L)\right)$, and thus

$$
\Delta_{\mathbb{Q}}\left(c_{1}(L)\right) \supseteq \operatorname{int}(\Delta(L)) \cap \mathbb{Q}^{n} .
$$




\section{Generalized Okounkov bodies}

From Theorem 4.8 we have $\Delta_{\mathbb{Q}}\left(c_{1}(L)\right) \subseteq \bigcup_{m=1}^{\infty} \frac{1}{m} \mu(m L)$; thus we get the inclusion

$$
\operatorname{int}(\Delta(L)) \cap \mathbb{Q}^{n} \subseteq \bigcup_{m=1}^{\infty} \frac{1}{m} \mu(m L),
$$

which means that all rational interior points of $\Delta(L)$ are valuative.

Pursuing the same philosophy as in Proposition 4.9, it is natural to extend results related to Okounkov bodies for big line bundles to the more general case of an arbitrary big class $\alpha \in H^{1,1}(X, \mathbb{R})$. We propose the following definition.

Definition 4.12. Let $X$ be a Kähler manifold of dimension $n$. We define the generalized Okounkov body of a big class $\alpha \in H^{1,1}(X, \mathbb{R})$ with respect to the fixed flag $Y \bullet$ by

$$
\Delta(\alpha)=\overline{\Delta_{\mathbb{R}}(\alpha)}=\overline{\Delta_{\mathbb{Q}}(\alpha)} .
$$

We have the following properties for generalized Okounkov bodies.

Proposition 4.13. Let $\alpha$ and $\beta$ be big classes, and let $\omega$ be any Kähler class. Then

(i) $\Delta(\alpha)+\Delta(\beta) \subseteq \Delta(\alpha+\beta)$,

(ii) $\operatorname{vol}_{\mathbb{R}^{n}}(\Delta(\omega))>0$.

(iii) $\Delta(\alpha)=\bigcap_{\epsilon>0} \Delta(\alpha+\epsilon \omega)$.

Proof. Part (i) is obvious from the definition of generalized Okounkov body. To prove part (ii), we use induction on the dimension. The result is obvious if $n=1$, assume now that part (ii) is true for $n-1$. We choose $t>0$ small enough that $\omega-t Y_{1}$ is still a Kähler class. By the main theorem of [CT14], any Kähler current $\left.T \in\left(\omega-t Y_{1}\right)\right|_{Y_{1}}$ with analytic singularities can be extended to a Kähler current $\widetilde{T} \in \omega-t Y_{1}$; thus we have

$$
\Delta(\omega) \bigcap t \times \mathbb{R}^{n-1}=t \times \Delta\left(\left.\left(\omega-t Y_{1}\right)\right|_{Y_{1}}\right),
$$

where $\Delta\left(\left.\left(\omega-t Y_{1}\right)\right|_{Y_{1}}\right)$ is the generalized Okounkov body of $\left.\left(\omega-t Y_{1}\right)\right|_{Y_{1}}$ with respect to the flag

$$
Y_{1} \supset Y_{2} \supset \cdots \supset Y_{n}=\{p\} .
$$

By induction, we have $\operatorname{vol}_{\mathbb{R}^{n-1}}\left(\Delta\left(\left.\left(\omega-t Y_{1}\right)\right|_{Y_{1}}\right)\right)>0$. Since $\Delta(\omega)$ contains the origin, we have $\operatorname{vol}_{\mathbb{R}^{n}}(\Delta(\omega))>0$.

Now we are ready to prove part (iii). By concavity, we have

$$
\Delta\left(\alpha+\epsilon_{1} \omega\right)+\Delta\left(\left(\epsilon_{2}-\epsilon_{1}\right) \omega\right) \subseteq \Delta\left(\alpha+\epsilon_{2} \omega\right)
$$

if $0 \leqslant \epsilon_{1}<\epsilon_{2}$. Since $\Delta(\omega)$ contains the origin, we have

$$
\Delta(\alpha) \subseteq \bigcap_{\epsilon>0} \Delta(\alpha+\epsilon \omega) \text { and } \Delta\left(\alpha+\epsilon_{1} \omega\right) \subseteq \Delta\left(\alpha+\epsilon_{2} \omega\right) .
$$

From the concavity property, we conclude that $\operatorname{vol}_{\mathbb{R}^{n}}(\Delta(\alpha+t \omega))$ is a concave function for $t \geqslant 0$, thus continuous. Then we have

$$
\operatorname{vol}_{\mathbb{R}^{n}}\left(\bigcap_{\epsilon>0} \Delta(\alpha+\epsilon \omega)\right)=\operatorname{vol}_{\mathbb{R}^{n}}(\Delta(\alpha))>0 .
$$

Since $\bigcap_{\epsilon>0} \Delta(\alpha+\epsilon \omega)$ and $\Delta(\alpha)$ are both closed and convex, we have

$$
\Delta(\alpha)=\bigcap_{\epsilon>0} \Delta(\alpha+\epsilon \omega) .
$$




\section{Y. DENG}

Remark 4.14. We do not know whether $\operatorname{vol}_{\mathbb{R}^{n}}(\Delta(\alpha))$ is independent of the choice of the admissible flag. However, in the next subsection we will prove that in the case of surfaces, we have

$$
\operatorname{vol}_{X}(\alpha)=2 \operatorname{vol}_{\mathbb{R}^{2}}(\Delta((\alpha)) ;
$$

in particular, the Euclidean volume of the generalized Okounkov body is independent of the choice of the flag. We conjecture that

$$
\operatorname{vol}_{\mathbb{R}^{n}}(\Delta(\alpha))=\frac{1}{n !} \cdot \operatorname{vol}_{X}(\alpha),
$$

as we proposed in the introduction.

\subsection{Generalized Okounkov bodies on complex surfaces}

Now, we will focus mainly on generalized Okounkov bodies of compact Kähler surfaces. In this section, $X$ denotes a compact Kähler surface. We henceforth fix an admissible flag

$$
X \supseteq C \supseteq\{x\}
$$

on $X$, where $C \subset X$ is an irreducible curve and $x \in C$ is a smooth point.

Definition 4.15. For any big class $\alpha \in H^{1,1}(X, \mathbb{R})$, we denote the restricted $\mathbb{R}$-convex body of $\alpha$ along $C$ by $\Delta_{\mathbb{R}, X \mid C}(\alpha)$. This is defined to be the set of Lelong numbers $\nu\left(\left.T\right|_{C}, x\right)$, where $T \in \alpha$ ranges over all the positive currents with analytic singularities such that $C \nsubseteq E_{+}(T)$. The restricted Okounkov body of $\alpha$ along $C$ is defined as

$$
\Delta_{X \mid C}(\alpha):=\overline{\Delta_{\mathbb{R}, X \mid C}(\alpha)} .
$$

When $\alpha=c_{1}(L)$ for some big line bundle $L$ on $X$, it is noticeable that $\Delta_{X \mid C}(\alpha)=\Delta_{X \mid C}(L)$, where $\Delta_{X \mid C}(L)$ is defined in [LM09]. When $L$ is ample, we have $\Delta_{X \mid C}(L)=\Delta\left(\left.L\right|_{C}\right)$. Indeed, it suffices to show that for any section $s \in H^{0}\left(C, \mathcal{O}_{C}(L)\right)$, there exists an integer $m$ such that $s^{\otimes m}$ can be extended to a section $S_{m} \in H^{0}\left(X, \mathcal{O}_{X}(m L)\right)$. This can be guaranteed by the Kodaira vanishing theorem. When $\alpha$ is any ample class, there is a similar theorem in [CT14] which shows that, for any Kähler current $\left.T \in \alpha\right|_{C}$ with mild analytic singularities, $T$ can be extended to a global Kähler current $\tilde{T} \in \alpha$. However, the proof in [CT14] is rather involved due to their general statement of the theorem. In the following proposition, we give a simple proof of the extension for Kähler currents when $X$ is a complex surface. The idea of the proof is borrowed from [CT14].

Proposition 4.16. If $\alpha$ is an ample class, then we have

$$
\Delta_{X \mid C}(\alpha)=\Delta\left(\left.\alpha\right|_{C}\right)=[0, \alpha \cdot C] .
$$

Proof. From Definition 4.15, we have $\Delta_{X \mid C}(\alpha) \subseteq \Delta\left(\left.\alpha\right|_{C}\right)$. It suffices to prove that for any Kähler current $\left.T \in \alpha\right|_{C}$ with mild analytic singularities, we have a positive current $\widetilde{T} \in \alpha$ with analytic singularities such that $\left.\widetilde{T}\right|_{C}=T$. First, we choose a Kähler form $\omega \in \alpha$. By assumption, we can write $T=\left.\omega\right|_{V}+d d^{c} \varphi$ for some quasi-plurisubharmonic function $\varphi$ on $C$ which has mild analytic singularities. Our goal is to extend $\varphi$ to a function $\Phi$ on $X$ such that $\omega+d d^{c} \Phi$ is a Kähler current with analytic singularities.

Choose $\epsilon>0$ small enough that

$$
T=\left.\omega\right|_{C}+i d d^{c} \varphi \geqslant 3 \epsilon \omega
$$

holds as currents on $C$. We can cover $C$ by finitely many charts $\left\{W_{j}\right\}_{1 \leqslant j \leqslant N}$ satisfying the following properties: 


\section{Generalized Okounkov Bodies}

(i) On each $W_{j}(j \leqslant k)$, there are local coordinates $\left(z_{1}^{(j)}, z_{2}^{(j)}\right)$ such that $C \cap W_{j}=\left\{z_{2}^{(j)}=0\right\}$ and

$$
\varphi=\frac{c_{j}}{2} \log \left|z_{1}^{(j)}\right|^{2}+g_{j}\left(z_{1}^{(j)}\right),
$$

where $g_{j}\left(z_{1}^{(j)}\right)$ is smooth and bounded on $W_{j} \cap C$. We denote the single pole of $T$ in $W_{j}(j \leqslant k)$ by $x_{j}$.

(ii) On each $W_{j}(j \leqslant k)$, the local potential $\varphi$ is smooth and bounded on $W_{j} \cap C$.

(iii) We have $x_{i} \notin \overline{W_{j}}$ for $i=1, \ldots, k$ and $j \neq i$.

Define a function $\varphi_{j}$ on $W_{j}$ (with analytic singularities) by

$$
\varphi_{j}\left(z_{1}^{(j)}, z_{2}^{(j)}\right)= \begin{cases}\varphi\left(z_{1}^{(j)}\right)+A\left|z_{2}^{(j)}\right|^{2} & \text { if } j>k, \\ \frac{c_{j}}{2} \log \left(\left|z_{1}^{(j)}\right|^{2}+\left|z_{2}^{(j)}\right|^{2}\right)+g_{j}\left(z_{1}^{(j)}\right)+A\left|z_{2}^{(j)}\right|^{2} & \text { if } j \leqslant k,\end{cases}
$$

where $A>0$ is a constant. If we shrink the charts $W_{j}$ slightly, still preserving the property that $C \subseteq \bigcup W_{j}$, we can choose $A$ sufficiently large that

$$
\omega+d d^{c} \varphi_{j} \geqslant 2 \epsilon \omega
$$

holds on $W_{j}$ for all $j$. We also need to construct slightly smaller open sets $W_{j}^{\prime} \Subset U_{j} \Subset W_{j}$ such that $\bigcup W_{j}^{\prime}$ is still a covering of $C$.

By construction, $\varphi_{j}$ is smooth when $j>k$, and $\varphi_{j}$ is smooth outside the log pole $x_{j}$ when $j \leqslant k$. By property (iii) above, we can glue the functions $\varphi_{j}$ together to produce a Kähler current

$$
\widetilde{T}=\left.\omega\right|_{U}+d d^{c} \widetilde{\varphi} \geqslant \epsilon \omega
$$

defined in a neighborhood $U$ of $C$ in $X$, thanks to Richberg's gluing procedure. Indeed, $\varphi_{i}$ is smooth on $W_{i} \cap W_{j}$ for any $j \neq i$, which is a sufficient condition for using the Richberg technique. From the construction of $\widetilde{\varphi}$, we know that $\left.\widetilde{\varphi}\right|_{C}=\varphi$ and that $\widetilde{\varphi}$ has log poles at every $x_{i}$ and is continuous outside $x_{1}, \ldots, x_{k}$.

On the other hand, since $\alpha$ is an ample class, there exists a rational number $\delta>0$ such that $\alpha-\delta\{C\}$ is still ample; thus we have a Kähler form $\omega_{1} \in \alpha-\delta\{C\}$. We can write $\omega_{1}+\delta[C]=$ $\omega+d d^{c} \phi$, where $\phi$ is smooth outside $C$, and for any point $x \in C$, we have

$$
\phi=\frac{\delta}{2} \log \left|z_{2}\right|^{2}+O(1)
$$

where $z_{2}$ is the local equation of $C$.

Since $\phi$ is continuous outside $C$, we can choose a large constant $B>0$ such that $\phi>\widetilde{\varphi}-B$ in a neighborhood of $\partial U$. Therefore, we define

$$
\Phi= \begin{cases}\max \{\widetilde{\varphi}, \phi+B\} & \text { on } U \\ \phi+B & \text { on } X-U,\end{cases}
$$

which is well defined on the whole of $X$ and satisfies $\omega+d d^{c} \Phi \geqslant \epsilon^{\prime} \omega$ for some $\epsilon^{\prime}>0$. Since $\phi=-\infty$ on $C$, while $\left.\widetilde{\varphi}\right|_{C}=\varphi$, it follows that $\left.\Phi\right|_{C}=\varphi$.

We claim that $\Phi$ also has analytic singularities. Around $x_{j}$, we have

$$
\begin{aligned}
\widetilde{\varphi}\left(z_{1}, z_{2}\right) & =\frac{c_{j}}{2} \log \left(\left|z_{1}\right|^{2}+\left|z_{2}\right|^{2}\right)+O(1), \\
\phi\left(z_{1}, z_{2}\right) & =\frac{\delta}{2} \log \left|z_{2}\right|^{2}+O(1),
\end{aligned}
$$




\section{Y. DENG}

for some local coordinates $\left(z_{1}, z_{2}\right)$ of $x_{j}$. Thus, locally we have

$$
\max \{\widetilde{\varphi}, \phi+A\}=\frac{1}{2} \log \left(\left|z_{1}\right|^{2 c_{j}}+\left|z_{2}\right|^{2 c_{j}}+\left|z_{2}\right|^{2 \delta}\right)+O(1) .
$$

Since $\Phi$ is continuous outside $x_{1}, \ldots, x_{k}$, our claim is proved.

LEMMA 4.17. Let $\alpha$ be a big and nef class on $X$; then for any $\epsilon>0$, there exists a Kähler current $T_{\epsilon} \in \alpha$ with analytic singularities such that the Lelong number satisfies $\nu\left(T_{\epsilon}, x\right)<\epsilon$ for any point in $X$. Moreover, $T_{\epsilon}$ also satisfies

$$
E_{+}(T)=E_{n K}(\alpha) .
$$

Proof. Since $\alpha$ is big, there exists a Kähler current with analytic singularities such that $E_{+}\left(T_{0}\right)=$ $E_{n K}(\alpha)$ and $T_{0}>\omega$ for some Kähler form $\omega$. Since $\alpha$ is also a nef class, for any $\delta>0$, there exists a smooth form $\theta_{\delta}$ in $\alpha$ such that $\theta_{\delta} \geqslant-\delta \omega$. Thus $T_{\delta}:=\delta T_{0}+(1-\delta) \theta_{\delta} \geqslant \delta^{2} \omega$ is a Kähler current with analytic singularities satisfying

$$
E_{+}\left(T_{\delta}\right)=E_{+}\left(T_{0}\right)=E_{n K}(\alpha) \text { and } \nu\left(T_{\delta}, x\right)=\delta \nu\left(T_{0}, x\right)
$$

for any $x \in X$. Since the Lelong number $\nu\left(T_{0}, x\right)$ is an upper-continuous function (thus bounded from above), $\nu\left(T_{\delta}, x\right)$ converges uniformly to 0 as $\delta$ tends to 0 . The lemma is proved.

Proposition 4.18. Let $\alpha$ be a big and nef class with $C \nsubseteq E_{n K}(\alpha)$. Then we have

$$
\Delta_{X \mid C}(\alpha)=\Delta\left(\left.\alpha\right|_{C}\right)=[0, \alpha \cdot C] .
$$

Proof. Assume $E_{n K}(\alpha)=\bigcup_{i=1}^{r} C_{i}$, where each $C_{i}$ is an irreducible curve. By Lemma 4.17, for any $\epsilon>0$ there exists a Kähler current $T_{\epsilon} \in \alpha$ with analytic singularities such that

$$
E_{+}\left(T_{\epsilon}\right)=E_{n K}(\alpha)=\operatorname{Null}(\alpha)=\bigcup_{i=1}^{r} C_{i}
$$

and $\nu\left(T_{\epsilon}, x\right)<\epsilon$ for all $x \in X$. Thus, the Siu decomposition

$$
T_{\epsilon}=R_{\epsilon}+\sum_{i=1}^{r} a_{i, \epsilon} C_{i}
$$

satisfies $0 \leqslant a_{i, \epsilon}<\epsilon$, and $R_{\epsilon}$ is a Kähler current whose analytic singularities are isolated points. By Remark 2.5, the cohomology class $\left\{R_{\epsilon}\right\}$ is a Kähler class and converges to $\alpha$ as $\epsilon \rightarrow 0$. In particular, $\left|\left\{R_{\epsilon}\right\} \cdot C-\alpha \cdot C\right|<A \epsilon$, where $A$ is a constant.

By Proposition 4.16, there exists a Kähler current $S_{\epsilon} \in\left\{R_{\epsilon}\right\}$ with analytic singularities such that $C \nsubseteq E_{+}\left(S_{\epsilon}\right)$ and $-\epsilon<\nu\left(\left.S_{\epsilon}\right|_{C}, x\right)-\left\{R_{\epsilon}\right\} \cdot C<0$. Thus $T_{\epsilon}^{\prime}:=S_{\epsilon}+\sum_{i=1}^{r} a_{i, \epsilon} C_{i}$ is a Kähler current in $\alpha$ with analytic singularities, and $-(1+A) \epsilon<\nu\left(\left.T_{\epsilon}^{\prime}\right|_{C}, x\right)-\alpha \cdot C$. Since $\alpha$ is big and nef, there exists a Kähler current $P_{\epsilon}$ in $\alpha$ with analytic singularities such that $\nu\left(\left.P_{\epsilon}\right|_{C}, x\right)<\epsilon$. Therefore, by the definition of $\Delta_{X \mid C}(\alpha)$ and the convexity property, we deduce that $[0, \alpha \cdot C] \subseteq$ $\Delta_{X \mid C}(\alpha)$. On the other hand, $\Delta_{X \mid C}(\alpha) \subseteq \Delta\left(\left.\alpha\right|_{C}\right)=[0, \alpha \cdot C]$ by definition. The proposition is proved.

Lemma 4.19. Let $\alpha$ be a big class on $X$ with divisorial Zariski decomposition $\alpha=Z(\alpha)+N(\alpha)$. Assume $C \nsubseteq E_{n K}(Z(\alpha))$, so that $C \nsubseteq \operatorname{Supp}(N(\alpha))$ by Theorem 2.14. Moreover, set

$$
f(\alpha)=\nu_{x}\left(\left.N(\alpha)\right|_{C}\right), \quad g(\alpha)=\nu_{x}\left(\left.N(\alpha)\right|_{C}\right)+Z(\alpha) \cdot C,
$$

where $\nu_{x}\left(\left.N(\alpha)\right|_{C}\right)=\nu\left(\left.N(\alpha)\right|_{C}, x\right)$. Then the restricted Okounkov body of $\alpha$ along $C$ is the interval

$$
\Delta_{X \mid C}(\alpha)=[f(\alpha), g(\alpha)]
$$




\section{Generalized Okounkov Bodies}

Proof. First, by Remark 2.8 we conclude that $T \mapsto T-N(\alpha)$ is a bijection between the positive currents in $\alpha$ and those in $Z(\alpha)$; thus we have

$$
E_{n K}(\alpha)=E_{n K}(Z(\alpha)) \bigcup \operatorname{supp}(N(\alpha))
$$

and

$$
C \not E_{n K}(Z(\alpha)) \Longleftrightarrow C \nsubseteq E_{n K}(\alpha)
$$

By the assumption of theorem, $\left.N(\alpha)\right|_{C}$ is a well-defined positive current with analytic singularities on $C$. By the definition of $\Delta_{\mathbb{R}, X \mid C}(\alpha)$, we have

$$
\Delta_{\mathbb{R}, X \mid C}(\alpha)=\Delta_{\mathbb{R}, X \mid C}(Z(\alpha))+\nu_{x}\left(\left.N(\alpha)\right|_{C}\right) .
$$

We take the closure of the sets to get

$$
\Delta_{X \mid C}(\alpha)=\Delta_{X \mid C}(Z(\alpha))+\nu_{x}\left(\left.N(\alpha)\right|_{C}\right) .
$$

Since $\alpha$ is big, $Z(\alpha)$ is big and nef, and by Proposition 4.18 we have

$$
\Delta_{X \mid C}(Z(\alpha))=[0, Z(\alpha) \cdot C] .
$$

We have proved the lemma.

Definition 4.20. If $\alpha$ is big and $\beta$ is pseudo-effective, then the slope of $\beta$ with respect to $\alpha$ is defined as

$$
s=s(\alpha, \beta)=\sup \{t>0 \mid \alpha-t \beta \text { is big }\} .
$$

Remark 4.21. Since the big cone is open, we know that $\{t>0 \mid \alpha>t \beta\}$ is an open set in $\mathbb{R}^{+}$. Thus $\alpha-s \beta$ belongs to the boundary of the big cone denoted by $\partial \mathcal{E}$, and $\operatorname{vol}_{X}(\alpha-s \beta)=0$.

Proof of Theorem 1.3. For $t \in[0, s)$, we put $\alpha_{t}=\alpha-t\{C\}$, and let $Z_{t}:=Z\left(\alpha_{t}\right)$ and $N_{t}:=N\left(\alpha_{t}\right)$ be the positive and negative parts of the divisorial Zariski decomposition of $\alpha_{t}$, respectively.

(i) First, we assume that $C$ is nef. By Theorem 2.14, the prime divisors in $E_{n K}\left(Z\left(\alpha_{t}\right)\right)$ form an exceptional family, thus $C \nsubseteq E_{n K}\left(Z\left(\alpha_{t}\right)\right)$ and $C \nsubseteq E_{n K}\left(\alpha_{t}\right)$ by (4.2). By Lemma 4.19 we have $\Delta_{X \mid C}\left(\alpha_{t}\right)=\left[\nu_{x}\left(\left.N_{t}\right|_{C}\right), Z_{t} \cdot C+\nu_{x}\left(\left.N_{t}\right|_{C}\right)\right]$.

By the definitions of the $\mathbb{R}$-convex body and restricted $\mathbb{R}$-convex body, we have

$$
\Delta_{\mathbb{R}}(\alpha) \bigcap t \times \mathbb{R}=t \times \Delta_{\mathbb{R}, X \mid C}\left(\alpha_{t}\right) .
$$

Thus

$$
t \times \overline{\Delta_{\mathbb{R}, X \mid C}\left(\alpha_{t}\right)} \subseteq \overline{\Delta_{\mathbb{R}}(\alpha)} \bigcap t \times \mathbb{R} .
$$

However, since both $\Delta_{\mathbb{R}, X}(\alpha)$ and $\Delta_{\mathbb{R}, X \mid C}\left(\alpha_{t}\right)$ are closed convex sets in $\mathbb{R}^{2}$ and $\mathbb{R}$, we have

$$
t \times \overline{\Delta_{\mathbb{R}, X \mid C}\left(\alpha_{t}\right)}=\overline{\Delta_{\mathbb{R}}(\alpha)} \bigcap t \times \mathbb{R} ;
$$

therefore

$$
t \times \Delta_{X \mid C}\left(\alpha_{t}\right)=\Delta(\alpha) \bigcap t \times \mathbb{R}
$$

Let

$$
f(t)=\nu_{x}\left(\left.N_{t}\right|_{C}\right), \quad g(t)=Z_{t} \cdot C+\nu_{x}\left(\left.N_{t}\right|_{C}\right) ;
$$

then $\Delta(\alpha) \bigcap[0, s) \times \mathbb{R}$ is the region bounded by the graphs of $f(t)$ and $g(t)$.

Now, we prove the piecewise linearity of $f(t)$ and $g(t)$. By Lemma 3.1, we have $N_{t_{1}} \leqslant N_{t_{2}}$ if $0 \leqslant t_{1} \leqslant t_{2}<s$; thus $f(t)$ is increasing. Since $N_{t}$ is an exceptional divisor by Theorem 2.15, the number of the prime components of $N_{t}$ is uniformly bounded by the Picard number $\rho(X)$. 


\section{Y. DENG}

Thus we can write $N_{t}=\sum_{i=1}^{r} a_{i}(t) N_{i}$, where $a_{i}(t) \geqslant 0$ is an increasing and continuous function. Moreover, there exist $t_{i}$ with $0=t_{0}<t_{1}<\cdots<t_{k}=s$ such that the prime components of $N_{t}$ are the same when $t$ lies in the interval $\left(t_{i}, t_{i+1}\right)$ for $i=0, \ldots, k-1$, and the number of prime components of $N_{t}$ will increase at every $t_{i}$ for $i=1, \ldots, k-1$. We write $s_{i}=\left(t_{i-1}+t_{i}\right) / 2$ for $i=1, \ldots, k$.

We denote the linear subspace of $H^{1,1}(X, \mathbb{R})$ spanned by the prime components of $N_{s_{i}}$ by $V_{i}$, and let $V_{i}^{\perp}$ be the orthogonal space of $V_{i}$ with respect to $q$. By the proof of Lemma 3.1, for $t \in\left(t_{i-1}, t_{i}\right)$ we have

$$
Z_{t}=Z_{s_{i}}+\left(s_{i}-t\right)\{C\}_{i}^{\perp}, \quad N_{t}=N_{s_{i}}+\left(s_{i}-t\right) C_{i}^{\|}
$$

where $\{C\}_{i}^{\perp}$ is the projection of $\{C\}$ on $V_{i}^{\perp}$, and $C_{i}^{\|}$is a linear combination of the prime components of $N_{s_{i}}$ satisfying that the cohomology class $\left\{C_{i}^{\|}\right\}$is equal to the projection of $\{C\}$ on $V_{i}$. By Theorem 2.14, the cohomology classes of prime components of $N_{s_{i}}$ are all independent, thus $C_{i}^{\|}$is uniquely defined. The piecewise linearity of $f(t)$ and $g(t)$ follows directly from (4.4), and thus $f(t)$ and $g(t)$ can be continuously extended to $s$. We conclude that $\Delta(\alpha)$ is the region bounded by the graphs of $f(t)$ and $g(t)$ for $t \in[0, s]$, and the vertices of $\Delta(\alpha)$ are contained in the set $\left\{\left(t_{i}, f\left(t_{i}\right)\right),\left(t_{j}, g\left(t_{j}\right)\right) \in \mathbb{R}^{2} \mid i, j=0, \ldots, k\right\}$. This means that a vertex of $\Delta(\alpha)$ may only occur for those $t \in[0, s]$ where a new curve appears in $N_{t}$. Since $r \leqslant \rho(X)$, the number of vertices is bounded by $2 \rho(X)+2$. The fact that $f(t)$ is convex and $g(t)$ concave is a consequence of the convexity of $\Delta(\alpha)$.

By (4.3), we have

$$
\begin{aligned}
2 \operatorname{vol}_{\mathbb{R}^{2}}(\Delta(\alpha)) & =2 \int_{0}^{s} \operatorname{vol}_{\mathbb{R}}\left(\Delta_{X \mid C}\left(\alpha_{t}\right)\right) \mathrm{d} t=2 \int_{0}^{s} Z_{t} \cdot C \mathrm{~d} t \\
& =\operatorname{vol}_{X}(\alpha)-\operatorname{vol}_{X}(\alpha-s C) \\
& =\operatorname{vol}_{X}(\alpha),
\end{aligned}
$$

where the second equality follows by Proposition 4.18, the third one by Theorem 3.2, and the last one by Remark 4.21. We have proved the theorem under the assumption that $C$ is nef.

(ii) Now, we prove the theorem when $C$ is not nef, that is, $C^{2}<0$. Recall that $a:=\sup \{t>$ $\left.0 \mid C \subseteq E_{n K}\left(\alpha_{t}\right)\right\}$. By (4.2), if $C \subseteq E_{n K}\left(\alpha_{t}\right)$ for some $t \in[0, s)$, we have $C \subseteq E_{n K}\left(Z\left(\alpha_{t}\right)\right)$. By the proof in Theorem 3.4, we have

$$
Z\left(\alpha_{\tau}\right) \cdot C=0, \quad Z\left(\alpha_{\tau}\right)=Z\left(\alpha_{t}\right)
$$

for $0 \leqslant \tau \leqslant t$. Thus we have

$$
\left\{0 \leqslant t<s \mid C \nsubseteq E_{n K}\left(\alpha_{t}\right)\right\}=(a, s),
$$

and $\Delta(\alpha)$ is contained in $[a, s] \times \mathbb{R}$. By Theorem 3.4, we also have

$$
\begin{aligned}
2 \operatorname{vol}_{\mathbb{R}^{2}}(\Delta(\alpha)) & =2 \int_{a}^{s} \operatorname{vol}_{\mathbb{R}}\left(\Delta_{X \mid C}\left(\alpha_{t}\right)\right) \mathrm{d} t=2 \int_{a}^{s} Z_{t} \cdot C \mathrm{~d} t \\
& =\operatorname{vol}_{X}\left(\alpha_{a}\right)-\operatorname{vol}_{X}\left(\alpha_{s}\right) \\
& =\operatorname{vol}_{X}(\alpha) .
\end{aligned}
$$

Since the prime component of $N_{t_{1}}$ is contained in that of $N_{t_{2}}$ if $a<t_{1} \leqslant t_{2}<s$, using the same arguments above, we obtain the piecewise linearity of $f(t)$ and $g(t)$, which can also be extended to $s$. The theorem is proved completely. 


\section{Generalized Okounkov Bodies}

Remark 4.22. If $X$ is a projective surface, then by the main result in [BKS04], the cone of big divisors of $X$ admits a locally finite decomposition into locally polyhedral subcones such that the support of the negative part in the Zariski decomposition is constant on each subcone. It is noticeable that if we only assume $X$ to be Kähler, this decomposition still holds if we replace the cone of big divisors by the cone of big classes and use divisorial Zariski decomposition instead. This property ensures that the generalized Okounkov bodies should also be polygons.

\subsection{Generalized Okounkov bodies for pseudo-effective classes}

Throughout this subsection, $X$ will stand for a Kähler surface if not explicitly mentioned otherwise. Our main goal in this subsection is to study the behavior of generalized Okounkov bodies on the boundary of the big cone.

Definition 4.23. Let $X$ be any Kähler manifold, and let $\alpha \in H^{1,1}(X, \mathbb{R})$ be any pseudo-effective class. We define the generalized Okounkov body $\Delta(\alpha)$ with respect to the fixed flag by

$$
\Delta(\alpha):=\bigcap_{\epsilon>0} \Delta(\alpha+\epsilon \omega)
$$

where $\omega$ is any Kähler class.

It is easy to check that our definition does not depend on the choice of $\omega$, and if $\alpha$ is big, then by Proposition 4.13, the definition is consistent with Definition 4.12. Now, we recall the definition of numerical dimension for any real $(1,1)$-class.

Definition 4.24. Let $X$ be a compact Kähler manifold. For a class $\alpha \in H^{1,1}(X, \mathbb{R})$, the numerical dimension $n(\alpha)$ is defined to be $-\infty$ if $\alpha$ is not pseudo-effective and

$$
n(\alpha)=\max \left\{p \in \mathbb{N},\left\langle\alpha^{p}\right\rangle \neq 0\right\}
$$

if $\alpha$ is pseudo-effective.

The right-hand side of the equation above involves the positive intersection product $\left\langle\alpha^{p}\right\rangle \in$ $H_{\geqslant 0}^{p, p}(X, \mathbb{R})$ defined in [BDPP13]. When $X$ is a Kähler surface, we simply have

$$
n(\alpha)=\max \left\{p \in \mathbb{N}, Z(\alpha)^{p} \neq 0\right\}, \quad p \in\{0,1,2\} .
$$

If $n(\alpha)=2$, then $\alpha$ is big and the situation is studied in the last subsection. Throughout this subsection, we assume $\alpha \in \partial \mathcal{E}$.

Lemma 4.25. Let $\left\{N_{1}, \ldots, N_{r}\right\}$ be an exceptional family of prime divisors, and let $\omega$ be any Kähler class. Then there exist unique positive numbers $b_{1}, \ldots, b_{r}$ such that $\omega+\sum_{i=1}^{r} b_{i} N_{i}$ is big and nef satisfying $\operatorname{Null}\left(\omega+\sum_{i=1}^{r} b_{i} N_{i}\right)=\bigcup_{i=1}^{r} N_{i}$.

Proof. If we set

$$
\left(\begin{array}{c}
b_{1} \\
\vdots \\
b_{r}
\end{array}\right)=-S^{-1} \cdot\left(\begin{array}{c}
\omega \cdot N_{1} \\
\vdots \\
\omega \cdot N_{r}
\end{array}\right),
$$

where $S$ denotes the intersection matrix of $\left\{N_{1}, \ldots, N_{r}\right\}$, we have $\left(\omega+\sum_{i=1}^{r} b_{i} N_{i}\right) \cdot N_{j}=0$ for $j=1, \ldots, r$. By Lemma 3.5, we conclude that all $b_{i}$ are positive and thus $\omega+\sum_{i=1}^{r} b_{i} N_{i}$ is big and nef. 


\section{Y. DENG}

Proposition 4.26. Let $\alpha$ be any pseudo-effective class with $N(\alpha)=\sum_{i=1}^{r} a_{i} N_{i}$, and let $\omega$ be a Kähler class. Then for $\epsilon>0$ small enough, we have the divisorial Zariski decomposition

$$
Z(\alpha+\epsilon \omega)=Z(\alpha)+\epsilon\left(\omega+\sum_{i=1}^{r} b_{i} N_{i}\right), \quad N(\alpha+\epsilon \omega)=\sum_{i=1}^{r}\left(a_{i}-\epsilon b_{i}\right) N_{i},
$$

where $b_{i}$ is the positive number defined in Lemma 4.25.

Proof. Since $Z(\alpha)+\epsilon\left(\omega+\sum_{i=1}^{r} b_{i} N_{i}\right)$ is nef and orthogonal to all $N_{i}$ by Lemma 4.25 , by Theorem 2.15, if $\epsilon$ satisfies $a_{i}-\epsilon b_{i}>0$ for all $i$, the divisorial decomposition in the proposition holds.

If $n(\alpha)=0$, we have $Z(\alpha)=0$, and thus $\alpha=\sum_{i=1}^{r} a_{i} N_{i}$ is an exceptional effective $\mathbb{R}$-divisor. We fix a flag

$$
X \supseteq C \supseteq\{x\},
$$

where $C \neq N_{i}$ for all $i$. We then have the following result.

Theorem 4.27. For any pseudo-effective class $\alpha$ with numerical dimension $n(\alpha)=0$, we have

$$
\Delta_{(C, x)}(\alpha)=0 \times \nu_{x}\left(\left.N(\alpha)\right|_{C}\right) .
$$

Proof. We assume $N(\alpha)=\sum_{i=1}^{r} a_{i} N_{i}$. Fix a Kähler class $\omega$. By Proposition 4.26, for $\epsilon$ small enough, we have

$$
Z(\alpha+\epsilon \omega)=\epsilon\left(\omega+\sum_{i=1}^{r} b_{i} N_{i}\right), \quad N(\alpha+\epsilon \omega)=\sum_{i=1}^{r}\left(a_{i}-\epsilon b_{i}\right) N_{i},
$$

where $b_{i}$ is the positive number defined in Lemma 4.25. Since $T \mapsto T-N(\alpha+\epsilon \omega)$ is a bijection between the positive currents in $\alpha+\epsilon \omega$ and those in $Z(\alpha+\epsilon \omega)$, we have

$$
\Delta(\alpha+\epsilon \omega)=\epsilon \Delta\left(\omega+\sum_{i=1}^{r} b_{i} N_{i}\right)+\nu\left(\sum_{i=1}^{r}\left(a_{i}-\epsilon b_{i}\right) N_{i}\right)
$$

where $\nu\left(\sum_{i=1}^{r}\left(a_{i}-\epsilon b_{i}\right) N_{i}\right)=\nu_{(C, x)}\left(\sum_{i=1}^{r}\left(a_{i}-\epsilon b_{i}\right) N_{i}\right)$ is the valuation-like function defined in Section 4.1. Thus the diameter of $\Delta(\alpha+\epsilon \omega)$ converges to 0 when $\epsilon$ tends to 0 , and we conclude that $\Delta(\alpha)$ is a single point in $\mathbb{R}^{2}$. Since

$$
\begin{aligned}
\Delta(\alpha+\epsilon \omega) \bigcap 0 \times \mathbb{R} & =0 \times \Delta_{X \mid C}(\alpha+\epsilon \omega) \\
& =0 \times\left[\nu_{x}\left(\left.N(\alpha+\epsilon \omega)\right|_{C}\right), \nu_{x}\left(\left.N(\alpha+\epsilon \omega)\right|_{C}\right)+Z(\alpha+\epsilon \omega) \cdot C\right],
\end{aligned}
$$

by (4.5) we have

$$
\Delta(\alpha) \bigcap 0 \times \mathbb{R}=0 \times \nu_{x}\left(\left.\sum_{i=1}^{r} a_{i} N_{i}\right|_{C}\right),
$$

and we have proved the first part of Theorem 1.8.

If $n(\alpha)=1$, then $Z(\alpha)$ is nef but not big. If there exists one irreducible curve $C$ such that $Z(\alpha) \cdot C>0$, we fix the flag

$$
X \supseteq C \supseteq\{x\}
$$

We then have the following result.

TheOREm 4.28. For any pseudo-effective class $\alpha$ with numerical dimension $n(\alpha)=1$, we have

$$
\Delta(\alpha)=0 \times\left[\nu_{x}\left(\left.N(\alpha)\right|_{C}\right), \nu_{x}\left(\left.N(\alpha)\right|_{C}\right)+Z(\alpha) \cdot C\right] .
$$




\section{Generalized OKounkov Bodies}

Proof. By the assumption $Z(\alpha) \cdot C>0$, we know that $C \nsubseteq \operatorname{Supp}(N(\alpha))$. By Proposition 4.26, when $\epsilon$ small enough, the divisorial Zariski decomposition for $\alpha+\epsilon \omega$ is

$$
Z(\alpha+\epsilon \omega)=Z(\alpha)+\epsilon\left(\omega+\sum_{i=1}^{r} b_{i} N_{i}\right), \quad N(\alpha+\epsilon \omega)=\sum_{i=1}^{r}\left(a_{i}-\epsilon b_{i}\right) N_{i},
$$

where $b_{i}$ is the positive number defined in Lemma 4.25. Consequently, we have

$$
\begin{aligned}
\Delta(\alpha) \bigcap 0 \times \mathbb{R} & =\bigcap_{\epsilon>0} \Delta(\alpha+\epsilon \omega) \bigcap 0 \times \mathbb{R} \\
& =\bigcap_{\epsilon>0} 0 \times\left[\nu_{x}\left(\left.N(\alpha+\epsilon \omega)\right|_{C}\right), \nu_{x}\left(\left.N(\alpha+\epsilon \omega)\right|_{C}\right)+Z(\alpha+\epsilon \omega) \cdot C\right] \\
& =0 \times\left[\nu_{x}\left(\left.\sum_{i=1}^{r} a_{i} N_{i}\right|_{C}\right), \nu_{x}\left(\left.\sum_{i=1}^{r} a_{i} N_{i}\right|_{C}\right)+Z(\alpha) \cdot C\right]
\end{aligned}
$$

Since we have

$$
\operatorname{vol}_{\mathbb{R}^{2}}(\Delta(\alpha))=\lim _{\epsilon \rightarrow 0} \operatorname{vol}_{\mathbb{R}^{2}}(\Delta(\alpha+\epsilon \omega))=\lim _{\epsilon \rightarrow 0} Z(\alpha+\epsilon \omega)^{2}=0
$$

and $\Delta(\alpha)$ is a closed convex set, we conclude that there are no points of $\Delta(\alpha)$ which lie outside $0 \times \mathbb{R}$ as $\operatorname{vol}_{\mathbb{R}}(\Delta(\alpha) \bigcap 0 \times \mathbb{R})=Z(\alpha) \cdot C>0$. This finishes the proof of Theorem 1.8.

\section{ACKNOWLEDGEMENTS}

I would like to express my warmest gratitude to my thesis supervisor Professor Jean-Pierre Demailly for his many valuable suggestions and help in this work. I also would like to thank Professor Sen $\mathrm{Hu}$ for his constant encouragement, and the anonymous referee for very carefully reading.

\section{REFERENCES}

BDPP13 S. Boucksom, J.-P. Demailly, M. Păun, and T. Peternell, The pseudo-effective cone of a compact Kähler manifold and varieties of negative Kodaira dimension, J. Algebraic Geom. 22 (2013), no. 2, 201-248; https://doi.org/10.1090/S1056-3911-2012-00574-8.

BFJ09 S. Boucksom, C. Favre, and M. Jonsson, Differentiability of volumes of divisors and a problem of Teissier, J. Algebraic Geom. 18 (2009), no. 2, 279-308; https://doi.org/10.1090/ S1056-3911-08-00490-6.

BKS04 T. Bauer, A. Küronya, and T. Szemberg, Zariski chambers, volumes, and stable base loci, J. reine angew. Math. 576 (2004), 209-233; https://doi.org/10.1515/crll.2004.090.

Bou02 S. Boucksom, On the volume of a line bundle, Internat. J. Math. 13 (2002), no. 10, 1043-1063; https://doi.org/10.1142/S0129167X02001575.

Bou04_, Divisorial Zariski decompositions on compact complex manifolds, Ann. Sci. Éc. Norm. Supér. (4) 37 (2004), no. 1, 45-76; https://doi.org/10.1016/j.ansens.2003.04.002.

CT14 T. C. Collins and V. Tosatti, An extension theorem for Kähler currents with analytic singularities, Ann. Fac. Sci. Toulouse Math. (6) 23 (2014), no. 4, 893-905; https://doi.org/10.5802/ afst. 1429.

CT15 _ Kähler currents and null loci, Invent. Math. 202 (2015), no. 3, 1167-1198; https: //doi.org/10.1007/s00222-015-0585-9.

Dem92 J.-P. Demailly, Regularization of closed positive currents and intersection theory, J. Algebraic Geom. 1 (1992), no. 3, 361-409. 


\section{Y. DENG}

KK12a K. Kaveh and A. G. Khovanskii, Newton-Okounkov bodies, semigroups of integral points, graded algebras and intersection theory, Ann. of Math. 176 (2012), no. 2, 925-978; https://doi.org/ 10.4007/annals.2012.176.2.5.

KK12b_ Convex bodies associated to actions of reductive groups, Mosc. Math. J. 12 (2012), no. 2, 369-396.

KL14 A. Küronya and V. Lozovanu, Local positivity of linear series on surfaces, 2014, arXiv:1411.6205.

KLM12 A. Küronya, V. Lozovanu, and C. Maclean, Convex bodies appearing as Okounkov bodies of divisors, Adv. Math. 229 (2012), no. 5, 2622-2639; https://doi.org/10.1016/j.aim. 2012. 01.013.

Lam99 A. Lamari, Courants kählériens et surfaces compactes, Ann. Inst. Fourier (Grenoble) 49 (1999), no. 1, 263-285; https://doi.org/10.5802/aif.1673.

LM09 R. Lazarsfeld and M. Mustaţă, Convex bodies associated to linear series, Ann. Sci. Éc. Norm. Supér. (4) 42 (2009), no. 5, 783-835.

Oko96 A. Okounkov, Brunn-Minkowski inequality for multiplicities, Invent. Math. 125 (1996), no. 3, 405-411; https://doi.org/10.1007/s002220050081.

Pop16 D. Popovici, Sufficient bigness criterion for differences of two nef classes, Math. Ann. 364 (2016), no. 1, 649-655; https://doi.org/10.1007/s00208-015-1230-z.

Siu74 Y.T. Siu, Analyticity of sets associated to Lelong numbers and the extension of closed positive currents, Invent. Math. 27 (1974), 53-156; https://doi.org/10.1007/BF01389965.

Xia13 J. Xiao, Weak transcendental holomorphic Morse inequalities on compact kähler manifolds, 2013, arXiv:1308.2878.

Ya Deng Ya.Deng@ujf-grenoble.fr

Institut Fourier, Université Grenoble Alpes, 100 rue des Maths, 38610 Gières, France

School of Mathematical Sciences, University of Science and Technology of China, 230026 Hefei, China 